\title{
Analytical Footprints: Compact Representation of Elementary Singularities in Wavelet Bases
}

\author{
Dimitri Van De Ville, Member, IEEE, Brigitte Forster-Heinlein, Member, IEEE, Michael Unser, Fellow, IEEE, and \\ Thierry Blu, Senior Member, IEEE
}

\begin{abstract}
We introduce a family of elementary singularities that are point-Hölder $\alpha$-regular. These singularities are self-similar and are the Green functions of fractional derivative operators; i.e., by suitable fractional differentiation, one retrieves a Dirac $\delta$ function at the exact location of the singularity. We propose to use fractional operator-like wavelets that act as a multiscale version of the derivative in order to characterize and localize singularities in the wavelet domain. We show that the characteristic signature when the wavelet interacts with an elementary singularity has an asymptotic closed-form expression, termed the analytical footprint. Practically, this means that the dictionary of wavelet footprints is embodied in a single analytical form. We show that the wavelet coefficients of the (nonredundant) decomposition can be fitted in a multiscale fashion to retrieve the parameters of the underlying singularity. We propose an algorithm based on stepwise parametric fitting and the feasibility of the approach to recover singular signal representations.
\end{abstract}

Index Terms-Elementary singularities, footprints, fractional derivatives, generalized fractional splines, wavelet bases.

\section{INTRODUCTION}

W AVELET bases provide an elegant decomposition of $L^{2}(\mathbb{R})$, the space of square integrable functions [1], [2]. Various applications take advantage of the wavelet representation; e.g., data compression, denoising, and analysis of singularities [3]. For a biorthogonal wavelet system, it is well known that the order of approximation of the scaling function at the synthesis side imposes the number of vanishing moments-and thus the differentiation order - of the wavelet at the analysis side. The differentiator behavior has direct consequences for applications. First, transient features such as discontinuities are characterized by wavelet coefficients in their neighborhood only, which is

Manuscript received January 25, 2010; accepted August 02, 2010. Date of publication August 19, 2010; date of current version November 17, 2010. This work was supported in part by the Swiss National Science Foundation (Grants PP00P2-123438 and 200020-121763), and in part by the Center for Biomedical Imaging (CIBM) of the Geneva and Lausanne Universities, EPFL, and the Leenaards and Louis-Jeantet foundations. The associate editor coordinating the review of this manuscript and approving it for publication was Dr. Ivan W. Selesnick.

D. Van De Ville is with the Institute of Bioengineering, Ecole Polytechnique Fédérale de Lausanne (EPFL), Lausanne 1015, Switzerland and the Department of Radiology and Medical Informatics, University of Geneva, Geneva Switzerland (e-mail: dimitri.vandeville@epfl.ch).

B. Forster-Heinlein is with the Technische Universität München, 85747 Garching, Germany.

M. Unser is with the Biomedical Imaging Group (BIG), Ecole Polytechnique Fédérale de Lausanne (EPFL), Lausanne 1015, Switzerland.

T. Blu is with the Chinese University of Hong Kong (CUHK), Shatin, Hong Kong.

Color versions of one or more of the figures in this paper are available online at http://ieeexplore.ieee.org.

Digital Object Identifier 10.1109/TSP.2010.2068295 consistent with the derivative-like behavior. Second, the smooth parts of the signal get filtered out and transfered to the lowpass subband that is subsampled coarsely. Mallat and colleagues [4]-[6] used wavelets to recover a characteristic representation of the signal from its wavelet coefficients' zero-crossings (when the wavelet acts like a second-order derivative) or their modulus maxima (when it acts like a first-order derivative). Due to the lack of shift-invariance of the wavelet transform, Mallat and Zhong [5] proposed a redundant and nonorthogonal version, thus extending wavelet bases to wavelet frames. This transform was later also studied by Wang [7]. While the redundant wavelet transform makes the analysis of the coefficients easier, it brings along an important disadvantage, next to redundancy, which is the nonuniqueness of the synthesis step.

Description in terms of elementary singularities (e.g., spikes, jumps, or discontinuities of the derivatives) is one way to model signals. Mathematically, the behavior of elementary singularities can be characterized by their Lipschitz (or Hölder) exponent $\alpha$, which can be infered from the rate of decay of its wavelet coefficients as the scale $s$ decreases:

$$
\left|\left\langle f(\cdot), \sqrt{s^{-1}} \psi(\cdot / s-k)\right\rangle\right| \leq A s^{\alpha+1 / 2} .
$$

It also is the key property behind the concept of the cone of influence of a singularity over scale [6]. Modeling in terms of singularities is especially relevant for the field of seismic exploration, where the detection of different layers in the underground can be formulated as a "sparse spike deconvolution" problem [3, Sec. 13.3.2]. Here, the underlying signal is assumed to be a sum of spikes, measured through the seismic wavelet. In that case, it is appropriate to impose the sparsity constraint using $\ell^{1}$ minimization on the signal representation in the Dirac basis [8], [9]. The spike model can also be extended; for example, the Lipschitz exponent has been obtained using the continuous wavelet transform in order to measure local sharpness of the reflectors [10] or by investigating the effect of the singularity on the continuous wavelet [11]. This type of approach has also been pursued using discrete B-spline bases that are tuned to a large class of singularities [12]. Singularities and their multiscale properties have been used in other fields as well such as for the detection of characteristic points in ECG signals [13] or to perform step detection and estimation using multiscale products [14]. More recently, Dragotti and Vetterli proposed "wavelet footprints" as atoms that explicitly characterize signal jumps and that constitute a dictionary [15]; these footprints need to be learned from wavelet decompositions and become shift-variant in the case of wavelet bases. The requirement of learning the wavelet footprint comes from the fact that the 
mother wavelet of a discrete wavelet transform is often only known implicitly and does not have a tractable analytical expression (e.g., Daubechies' wavelet are defined by the iterated filter relation). Bruni and Vitulano proposed a practical scheme for more general singularities that is also capable of resolving overlapping footprints [16].

In this paper, we do not consider an explicit dictionary, but we derive a closed-form analytical footprint of the elementary singularity in a well-chosen wavelet basis-retrieving the singularities from their wavelet decomposition then becomes a parametric fitting problem. As a starting point, we consider the fractional derivative/integral operator $\partial_{\tau}^{\gamma}$, where the order $\gamma$ and the phase $\tau$ can both be real-valued. Elementary singularities are then introduced as the Green functions $\rho$ of the derivative operator $\partial_{\tau}^{\gamma}$, for $\gamma \geq 0$; i.e., they satisfy $\partial_{\tau}^{\gamma}\{\rho\}=\delta$. Equivalently, they can be interpreted as the impulse response of the fractional integral operator $\partial_{\tau}^{-\gamma}$. Next, we consider the family of semi-orthogonal fractional B-spline wavelet bases [17], [18] associated to $\partial_{\tau}^{\gamma}, \gamma \geq 1$, which have also been used for layer detection in seismic exploration [12]. We focus on a particular wavelet within this family, termed the "operator wavelet," for which we derive an explicit closed-form expression together with its fractional integrals that model the interaction of the wavelet with an elementary singularity. This allows us to generate the atoms of a dictionary without explicit learning; i.e., our analytical footprint contains all the information. The operator wavelet also has the attractive property that the wavelet coefficients within a basis decomposition of these singularities are mono- or bimodal only.

This paper is organized as follows. In Section II, we start by revisiting fractional derivatives and the associated singularities. Then, in Section III, we propose the design procedure for the operator wavelet and we investigate its main properties. We also characterize the wavelet and its fractional integrals, which leads to the definition of the analytical wavelet footprint. In Section IV, we demonstrate how to use these footprints with a practical stepwise parametric fitting algorithm that properly separates the singular and smooth parts of a signal.

Notations: We define the conventional inner product between two $L_{2}$ functions $f_{1}$ and $f_{2}$ as

$$
\left\langle f_{1}, f_{2}\right\rangle=\int_{-\infty}^{\infty} f_{1}(x) f_{2}^{*}(x) \mathrm{d} x
$$

where * denotes the complex conjugate. The associated Euclidean norm is $\|f\|_{L_{2}}^{2}=\langle f, f\rangle$. The Fourier transform of $f$ is defined as

$$
\hat{f}(\omega)=\int_{-\infty}^{\infty} f(x) e^{-j \omega x} \mathrm{~d} x .
$$

For sequences in $\ell_{2}$, we define the inner product

$$
\left\langle h_{1}, h_{2}\right\rangle_{\ell_{2}}=\sum_{k \in \mathbb{Z}} h_{1}(k) h_{2}^{*}(k) .
$$

The $z$-transform of a sequence $h \in \ell_{2}$ is denoted by

$$
H(z)=\sum_{k \in \mathbb{Z}} h[k] z^{-k}
$$

while the corresponding Fourier transform is $H\left(e^{j \omega}\right)$. We define the fractional power of a complex variable $z$ as $z^{\alpha}=|z|^{\alpha} e^{j \alpha \arg (z)}$ with $j=\sqrt{-1}$ and $\arg (z) \in[-\pi, \pi[$. When we omit the range for integrals and summations, they should be understood as over $\mathbb{R}$ and $\mathbb{Z}$, respectively.

\section{Fractional DeRIVATIVES AND BBELEMENTARY SINGULARITIES}

Fractional derivative/integral operators are a long-standing research topic in mathematics [19]-[22] with many applications in physics, for example [23]. These operators can be introduced in various ways [24], [25]; here, we define them in the sense of distributions by the Fourier-domain formulation:

$$
\partial_{\tau}^{\gamma} f(x)=\int_{-\infty}^{\infty} \underbrace{(-j \omega)^{\frac{\gamma}{2}-\tau}(j \omega)^{\frac{\gamma}{2}+\tau}}_{=::_{\tau}^{\gamma}(\omega)} \hat{f}(\omega) e^{j \omega x} \frac{\mathrm{d} \omega}{2 \pi}
$$

where $\gamma \in \mathbb{R}$ is the order and $\tau \in \mathbb{R}$ is the phase parameter. We also notice that the Fourier representation of the time-reversed (or adjoint) operator $\bar{\partial}_{\tau}^{\gamma}=\partial_{-\tau}^{\gamma}$ corresponds to the $\hat{\partial}_{\tau}^{\gamma}(-\omega)$. The fractional derivative/integral operators form a complete family of scale-invariant convolution operators [26].

Definition 1 (Elementary Singularity): The elementary singularity $\rho_{\tau}^{\gamma}(x)$ of order $\gamma$ and phase shift $\tau$ is the Green function of the operator $\partial_{\tau}^{\gamma}$ :

$$
\partial_{\tau}^{\gamma} \rho_{\tau}^{\gamma}(x)=\delta(x)
$$

These Green functions can be determined by inverse Fourier transformation and are given by

$$
\rho_{\tau}^{\gamma+1}(x)= \begin{cases}C_{\tau}^{\gamma}|x|^{\gamma}+D_{\tau}^{\gamma}|x|^{\gamma} \log |x|, & \gamma \text { is odd } \\ C_{\tau}^{\gamma}|x|^{\gamma} \log |x|+D_{\tau}^{\gamma}|x|^{\gamma} \operatorname{sign}(x), & \gamma \text { is even } \\ C_{\tau}^{\gamma}|x|^{\gamma}+D_{\tau}^{\gamma}|x|^{\gamma} \operatorname{sign}(x), & \text { otherwise }\end{cases}
$$

where $C_{\tau}^{\gamma}$ and $D_{\tau}^{\gamma}$ are suitable constants [18], [22, pp. 16-17], [27, ch. 2]-[29].

The prototypical example is the unit step function $u(x)=$ $\rho_{1 / 2}^{1}=(x)_{+}^{0}$, which is such that $\mathrm{D} u(x)=\delta(x)$, where $\mathrm{D}=$ $\partial_{1 / 2}^{1}$ is the ordinary derivative operator. Thanks to the scale-invariance of the associated operators, each Green function is self-similar in the sense that $\rho_{\tau}^{\gamma}(x / T)=\lambda \rho_{\tau}^{\gamma}(x)$ with $\lambda=$ $T^{1-\gamma}$. In Fig. 1, we show several subsets of our family of elementary singularities. For first-order singularities, they vary from the unit step to the spike, as shown in (a). Higher-order singularities characterize discontinuities of a corresponding $\gamma$ th order fractional derivative. Notice that the signal $\rho_{\tau}^{\gamma}$ has precisely one singularity at the origin with a Lipschitz exponent $\gamma-1$ [17].

Definition 2 (Singular Signal Model): A signal is said to be singular if it is a finite sum of weighted elementary singularities plus a smooth term that is $C^{\infty}$.

Specifically, we express a singular signal containing $M$ singularities of orders $\gamma_{m} \geq 0$, and with phase parameters $\tau_{m}$ as a sum of the elementary singularities $\rho_{\tau_{m}}^{\gamma_{m}}$ and the smooth part $f_{0}(x)$ :

$$
f(x)=\sum_{m=1}^{M} a_{m} \rho_{\tau_{m}}^{\gamma_{m}}\left(x-x_{m}\right)+f_{0}(x)
$$


(a)

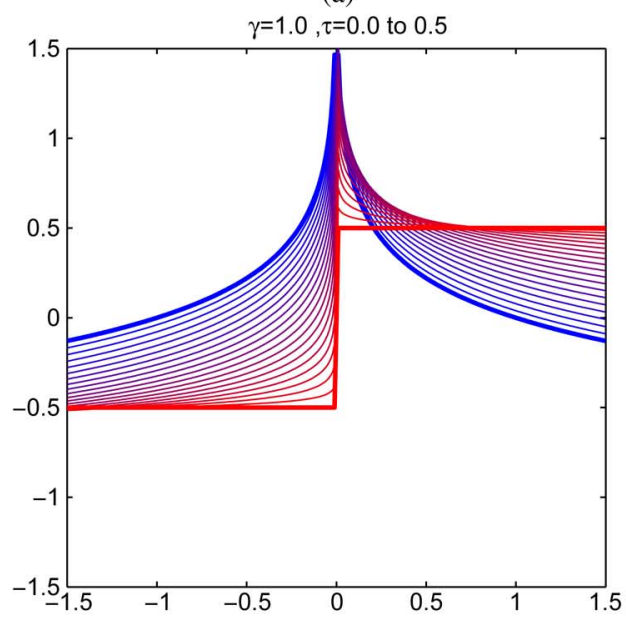

(c)

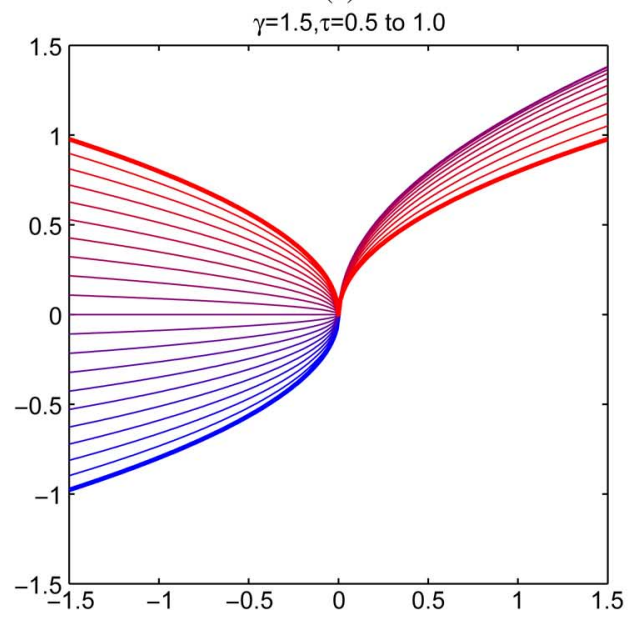

(b)

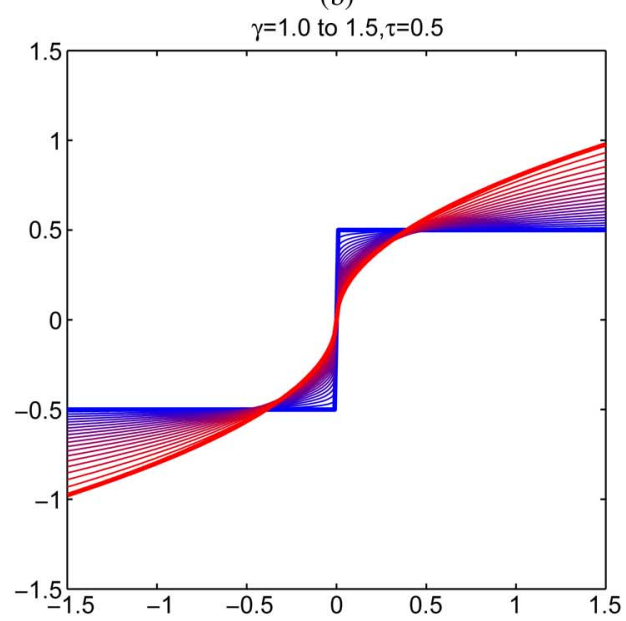

(d)

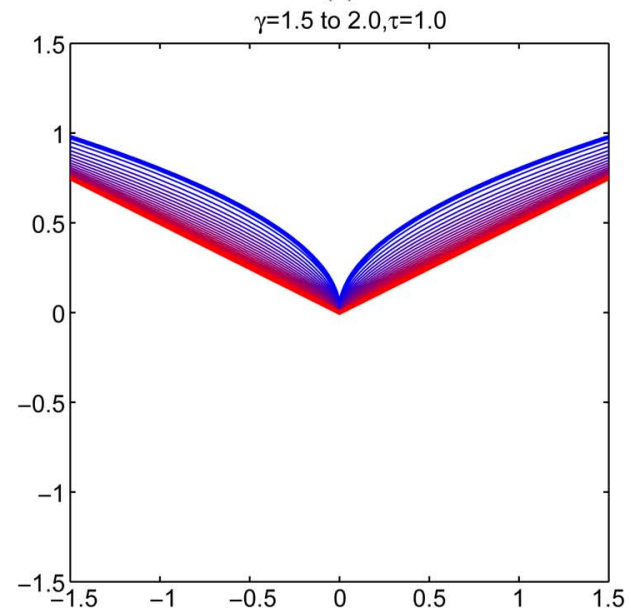

Fig. 1. Family of elementary singularities associated to the fractional derivative operator $\partial_{\tau}^{\gamma}$. Several subsets of singularities are shown in (a)-(d). The sweepthrough starts in blue and ends in red.

where the continuously-defined parameters are the amplitudes $a_{m}, m=1, \ldots, M$, and the positions $x_{m} \in \mathbb{R}$.

\section{OPERATOR WAVELETS}

\section{A. Fractional $(\alpha, \tau)$-Splines and Wavelets}

Closely related to the fractional derivative operators are the fractional B-splines of degree $\alpha \geq 0(\alpha \in \mathbb{R})$ and phase parameter $\tau \in \mathbb{R}$. These are most conveniently defined in the Fourier domain as

$$
\hat{\beta}_{\tau}^{\alpha}(\omega)=\left(\frac{1-e^{j \omega}}{-j \omega}\right)^{\frac{\alpha+1}{2}-\tau}\left(\frac{1-e^{-j \omega}}{j \omega}\right)^{\frac{\alpha+1}{2}+\tau} .
$$

This family of B-splines contains the traditional symmetric $(\tau=0)$ and causal $(\tau=(\alpha+1) / 2)$ B-splines. The Fourier domain definition of the B-spline can also be written as

$$
\hat{\beta}_{\tau}^{\alpha}(\omega)=\frac{\hat{\Delta}_{\tau}^{\alpha+1}\left(e^{j \omega}\right)}{\hat{\partial}_{\tau}^{\alpha+1}(\omega)}
$$

where the numerator

$$
\hat{\Delta}_{\tau}^{\alpha+1}(z)=(1-z)^{\frac{\alpha+1}{2}-\tau}\left(1-z^{-1}\right)^{\frac{\alpha+1}{2}+\tau}
$$

is the $z$-transform of the fractional finite difference operator $\Delta_{\tau}^{\gamma}$ of order $\gamma$ (convergence limited to the unit circle $z=e^{j \omega}$ ). We can interpret the B-spline as the application of the localization operator $\Delta_{\tau}^{\alpha+1}$ to the elementary singularity; i.e., $\beta_{\tau}^{\alpha}=$ $\Delta_{\tau}^{\alpha+1} \rho_{\tau}^{\alpha+1}(x)[30]$.

The $(\alpha, \tau)$-splines satisfy the (dyadic) two-scale relation where the scaling filter $h[k]$ relates B-splines at two consecutive scales as

$$
\beta_{\tau}^{\alpha}\left(\frac{x}{2}\right)=\sqrt{2} \sum_{k} h[k] \beta_{\tau}^{\alpha}(x-k)
$$

whose $z$-transform is given by

$$
\begin{aligned}
H(z) & =2^{-\alpha-\frac{1}{2}} \frac{\hat{\Delta}_{\tau}^{\alpha+1}\left(z^{2}\right)}{\hat{\Delta}_{\tau}^{\alpha+1}(z)} \\
& =2^{-\alpha-\frac{1}{2}}(1+z)^{\frac{\alpha+1}{2}-\tau}\left(1+z^{-1}\right)^{\frac{\alpha+1}{2}+\tau} \\
& =2^{-\alpha-\frac{1}{2}} \hat{\Delta}_{\tau}^{\alpha+1}(-z) .
\end{aligned}
$$

The autocorrelation filter of these splines is written in the Fourier domain as

$$
A\left(e^{j \omega}\right)=\sum_{k}\left|\hat{\beta}_{\tau}^{\alpha}(\omega+2 \pi k)\right|^{2} .
$$


The associated approximation spaces $\mathcal{V}_{i}$ at a (dyadic) scales $i \in \mathbb{Z}$ are specified as

$$
\mathcal{V}_{i}=\operatorname{span}\left\{\sqrt{2^{-i}} \beta_{\tau}^{\alpha}\left(2^{-i} x-k\right)\right\}_{k \in \mathbb{Z}}
$$

and the wavelet spaces

$$
\mathcal{W}_{i}=\operatorname{span}\left\{\sqrt{2^{-i}} \psi\left(2^{-i} x-k\right)\right\}_{k \in \mathbb{Z}}
$$

can be constructed as the orthogonal complement between two subsequent approximation spaces; i.e., $\mathcal{W}_{i} \oplus_{\perp} \mathcal{V}_{i}=\mathcal{V}_{i-1}$. The construction of a proper wavelet basis is summarized in the following proposition.

Proposition 1: For any given fractional B-spline $\beta_{\tau}^{\alpha}$, one can specify a corresponding semi-orthogonal spline wavelet $\psi$, parametrized by some bounded filter $Q(z)$. The following properties apply.

i) The wavelet is characterized by the relation

$$
\psi\left(\frac{x}{2}\right)=\sqrt{2} \sum_{k} w[k] \beta_{\tau}^{\alpha}(x-k)
$$

where $w[k]$ is the so-called wavelet filter. The Fourier domain counterpart is

$$
\hat{\psi}(2 \omega)=W\left(e^{j \omega}\right) \frac{\hat{\beta}_{\tau}^{\alpha}(\omega)}{\sqrt{2}}
$$

where $W(z)=z^{-1} Q\left(z^{2}\right) H\left(-z^{-1}\right) A(-z)$.

ii) The wavelet generates a Riesz basis whenever $Q\left(e^{j \omega}\right)$ is bounded by $C_{0} \leq\left|Q\left(e^{j \omega}\right)\right| \leq C_{1}$ for some positive constants $0<C_{0}, C_{1}<\infty$.

iii) At low frequencies, the wavelet follows the behavior of the associated derivative operator:

$$
\hat{\psi}(\omega) \propto \hat{\partial}_{\tau}^{\gamma}(-\omega) e^{-j \omega / 2}+O\left(|\omega|^{\gamma+1}\right), \quad \text { as } \omega \rightarrow 0 .
$$

For the proof see Appendix B.

Proposition 1 iii) shows that for any valid polynomial $Q\left(z^{2}\right)$, the corresponding wavelet behaves as a fractional derivative operator. Therefore, analyzing a signal $f(x)$ with this wavelet yields samples of the operator $\partial_{\tau}^{\gamma}$ applied to a smoothed version of the input signal:

$$
\langle f(\cdot), \psi(\cdot-k)\rangle=\partial_{\tau}^{\gamma}\{\phi * f\}(k)
$$

where the smoothing function is formally defined in the Fourier domain as $\hat{\phi}(\omega)=\hat{\psi}(\omega) / \hat{\partial}_{\tau}^{\gamma}(-\omega)$. The key point is that $\hat{\phi}(0)$ is well-defined and nonvanishing because of (19).

When $\alpha$ is integer, and $\tau=0$, one obtains the classical "symmetric B-spline wavelet" by setting $Q(z)=1$, which corresponds to the wavelet filter $W(z)$ with the shortest possible support [31]:

$$
W(z)=z^{-1} H\left(-z^{-1}\right) A(-z) .
$$

It is also possible to select $Q(z)$ to obtain the well-known (orthogonal) Battle-Lemarié wavelets [32].

\section{B. Operator Wavelets}

In this paper, we are interested in one particular fractional spline wavelet: the operator-like wavelet.
Theorem 1: The operator wavelet $\psi$ for the fractional B-spline scaling function of order $\gamma=\alpha+1$ and phase parameter $\tau$ corresponds to

$$
\psi\left(\frac{x}{2}\right)=\bar{\partial}_{\tau}^{\gamma} \beta_{\text {int }}^{2 \gamma-1}(x-1)
$$

where $\beta_{\mathrm{int}}^{2 \gamma-1}$ is the symmetric $2 \gamma$ th order interpolating B-spline. The operator wavelet has the following attractive properties.

i) It closely matches the derivative operator in the Fourier domain as shown by its Taylor development

$$
\hat{\psi}(\omega) \propto \hat{\partial}_{\tau}^{\gamma}(-\omega) e^{-j \omega / 2}+O\left(|\omega|^{3 \gamma}\right), \quad \operatorname{as} \omega \rightarrow 0
$$

which has a much smaller residual than (19).

ii) The wavelet and its fractional integrals have an asymptotic analytical form

$$
\partial_{-\tau_{0}}^{-\gamma_{0}} \psi(x) \rightarrow \underbrace{\frac{\pi^{\gamma-\gamma_{0}+1}}{4 \gamma} \operatorname{Re}\left[\frac{e^{j \pi\left(x-\left(\tau+\tau_{0}\right)\right)}}{\sin \left(\frac{\pi\left(\gamma-\gamma_{0}\right)}{4 \gamma}+j \frac{\pi^{2}(2 x-1)}{4 \gamma}\right)}\right]}_{g\left(x ; \gamma, \tau, \gamma_{0}, \tau_{0}\right)},
$$

for $x \rightarrow 0$, where $\gamma_{0}<\gamma$. The limiting form improves as the order $\gamma=\alpha+1$ increases.

For the proof, see Appendix C.

The form (22) of the operator wavelet deserves some further analysis. As the fractional derivative operator acts on the smoothing function that is an interpolating B-spline of twice of the order $\gamma$, the $\gamma$ th derivative of a spline of order $2 \gamma$ results in a spline of order $\gamma$, thus ensuring that the wavelet is contained in the span of the scaling function at the next finer scale. In fact, the operator wavelets coincide with the "cardinal spline wavelets" that were proposed by Chui and Wang [33] for causal B-spline of integer order. Here, we are proposing an extension to fractional operators (fractional orders and shifts). The fact that this construction improves the operator-like behavior of the wavelet does seem to have been emphasized before. The limiting form of the operator wavelet is also an original contribution of this work.

We should also note that there are other instances in the literature that are relying on an equation similar to (22), i.e., obtaining the wavelet by applying the operator to the interpolant of the "augmented order" function space, especially in higher dimensions. Micchelli et al. [34] proposed this construction for polyharmonic wavelets in any number of dimensions and for dyadic subsampling; these wavelets are related to the (iterated) Laplacian operator. This concept of wavelet design has also been generalized for almost any differential operator [35], including for Wirtinger-type operators [36], [37] and Riesz transforms [38].

Coming back to the fractional splines, we now show some examples where we compare the B-spline wavelets and the operator ones. In these comparisons, we assume that both wavelets are scaled in the same way. For these illustrations, we rescale the B-spline wavelet such that the frequency response for $\omega \rightarrow 0$ matches the derivative operator.

We start by noting that the B-spline wavelets and operator wavelets coincide for $\alpha=0$. In Fig. 2(a), we show the frequency response of both wavelets together with that of the corresponding derivative operator. For $\alpha>0$, the operator wavelet 
(a)

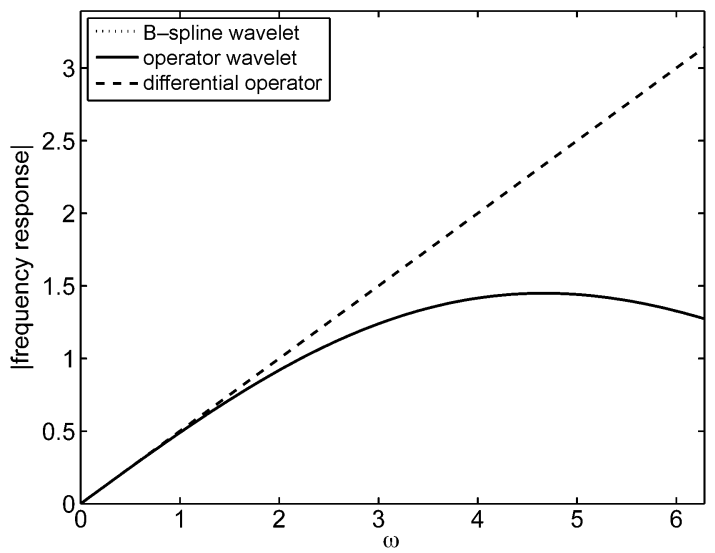

(c)

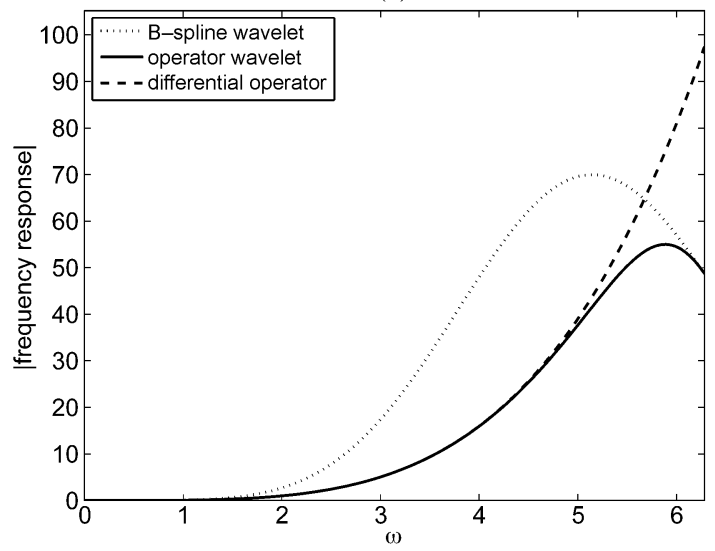

(b)

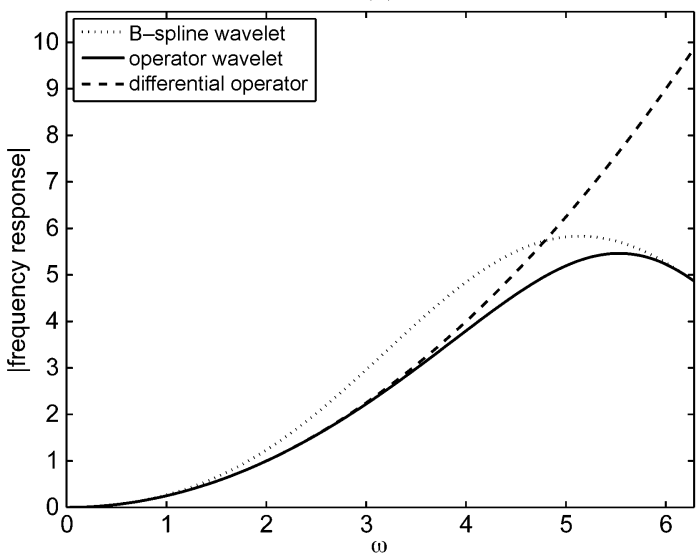

(d)

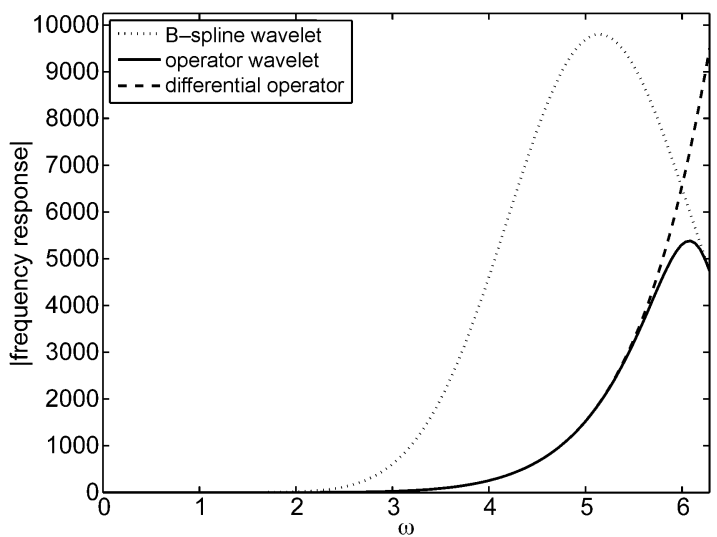

Fig. 2. Associated to the operator $\partial_{\tau}^{\gamma}$, modulus of the frequency response of the (rescaled) B-spline wavelet, the operator wavelet, and the derivative operator, for various values of $\gamma$. (a) $\gamma=1$; (b) $\gamma=2$; (c) $\gamma=4$; (d) $\gamma=8$.

matches the operator behavior more closely than the B-spline wavelet does. The examples in Fig. 2(b)-(d) illustrate how the operator wavelets coincide with the pure derivative operator in the Fourier domain over a large range of $\omega$. These plots hold for every shift $\tau$. It is also interesting to plot the wavelets in the spatial domain. In Fig. 3(a), the symmetric wavelets for $\alpha=1$ (i.e., the "linear" wavelets) are shown. The operator wavelet has a theoretical infinite support but stays in practice very similar to the B-spline wavelet. For higher degrees, such as $\alpha=2.5$ as shown Fig. 3(b), the difference between both wavelets becomes more significant.

\section{Filter Bank Implementation}

As usual, we introduce the subscript notations for the scaling function and wavelets as $\varphi_{i, n}(x)=2^{-i / 2} \varphi\left(x / 2^{i}-n\right)$ and $\psi_{i, n}(x)=2^{-i / 2} \psi\left(x / 2^{i}-n\right)$, respectively. Each signal $f(x)$ of $\mathcal{V}_{0}$ can be expanded uniquely as a sum of two parts coming from $\mathcal{V}_{1}$ and $\mathcal{W}_{1}$. Iterating the expansion $J$ times results into the decomposition

$$
f(x)=\sum_{n \in \mathbb{Z}} c_{(J)}[n] \varphi_{J, n}(x)+\sum_{i=1}^{J} \sum_{n \in \mathbb{Z}} d_{(i)}[n] \psi_{i, n}(x)
$$

where the coefficients $c_{(i)}[n]$ and $d_{(i)}[n]$ are determined as the projection of the signal in the subspaces; i.e., we have $c_{(i)}[n]=$ $\left\langle f, \tilde{\varphi}_{i, n}\right\rangle$ and $d_{(i)}[n]=\left\langle f, \tilde{\psi}_{i, n}\right\rangle$, where $\tilde{\varphi}$ and $\tilde{\psi}$ are the dual scaling function and dual wavelet. The dual function $\tilde{\varphi}$ is the unique function in $\mathcal{V}_{0}$ that satisfies $\left\langle\tilde{\varphi}_{0, k}, \varphi_{0, l}\right\rangle=\delta_{k-l}$.

In practice, the wavelet decomposition algorithm can be translated into an efficient filter bank implementation using the scaling and wavelet filters. Fig. 4 shows one iteration of the wavelet decomposition from both the point of view of the continuous domain and the discrete domain. To have the operator-like behavior on the input signal, one should put the operator wavelet as the wavelet $\tilde{\psi}$ at the analysis side, and its dual at the synthesis side. In that case, the filters of Fig. 4 are

$$
\begin{aligned}
& \tilde{G}_{0}(z)=H(z) \\
& G_{0}(z)=H(z) \frac{A(z)}{A\left(z^{2}\right)} \\
& \tilde{G}_{1}(z)=z^{-1} 2^{\alpha} \frac{H\left(-z^{-1}\right)}{A(z)} \\
& G_{1}(z)=z^{-1} 2^{-\alpha} H\left(-z^{-1}\right) \frac{A(z) A(-z)}{A\left(z^{2}\right)} .
\end{aligned}
$$

To correctly perform a wavelet decomposition of a signal $f(x)$, specified by its measurements $f(n)$ at the initial scale, one should apply a prefilter to obtain the coefficients $c_{(0)}[n]$ for its representation in $\mathcal{V}_{0}$. Here, we use the interpolation presentation of the B-spline representation at the initial scale. 
(a)

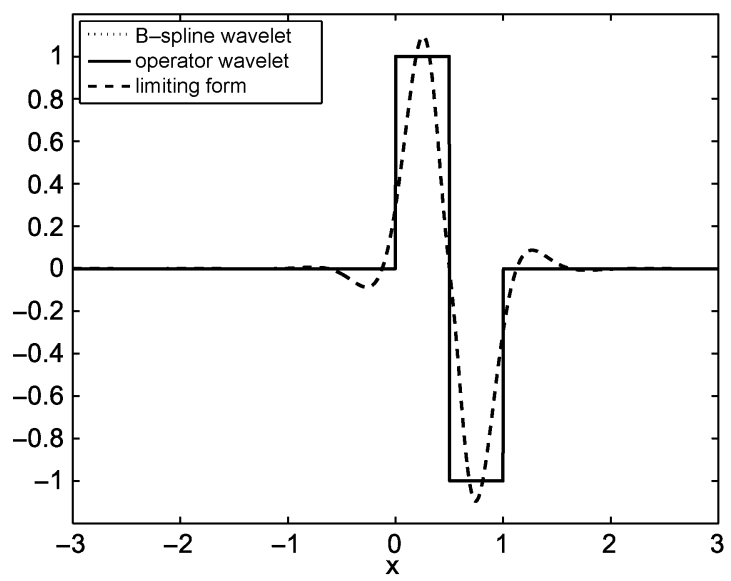

(c)

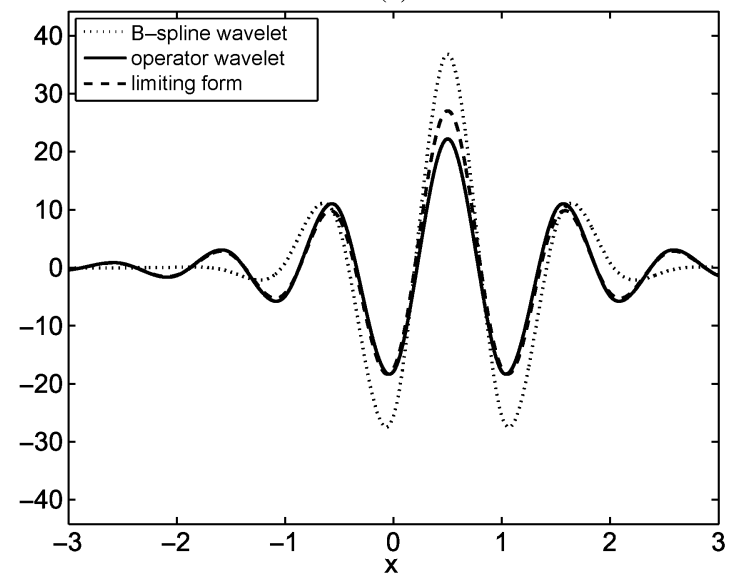

(b)

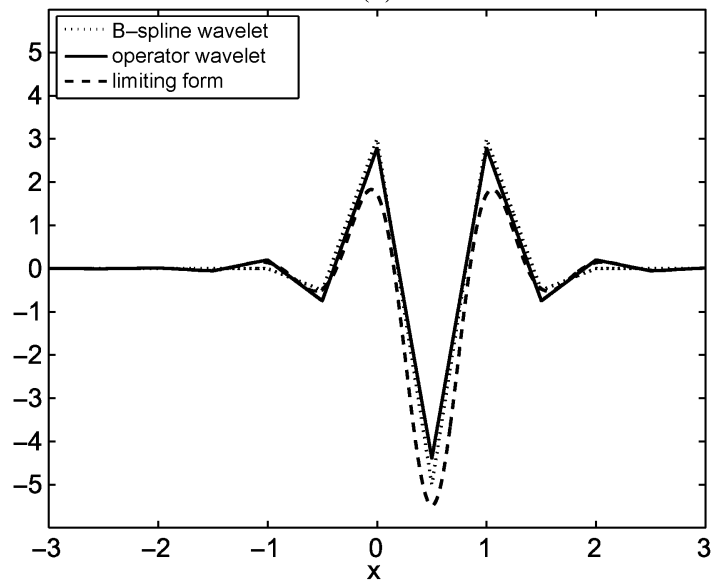

(d)

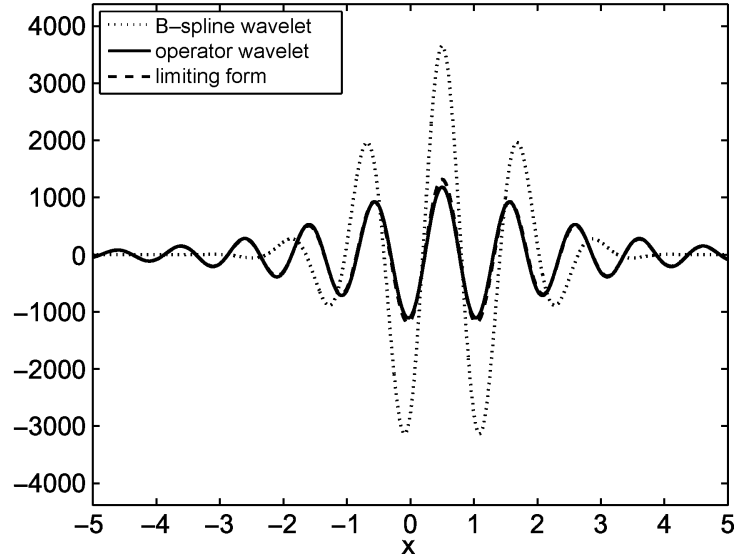

Fig. 3. The (rescaled) B-spline wavelet, the operator wavelet, and the limiting form, for various values of $\gamma$ associated to the operator $\partial_{\tau}^{\gamma}$, with $\tau=\gamma / 2$. (a) $\gamma=1$; (b) $\gamma=2$; (c) $\gamma=4$; (d) $\gamma=8$.

(a)

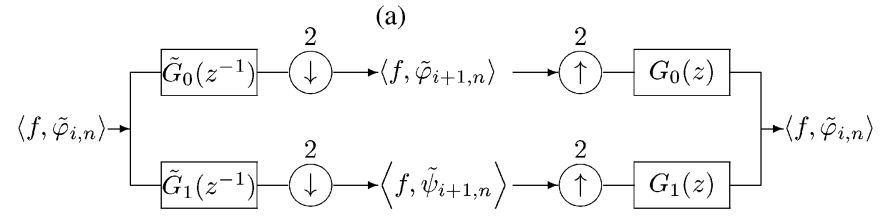

(b)

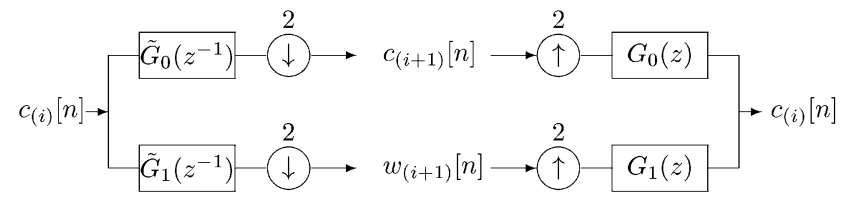

Fig. 4. The discrete wavelet transform for one decomposition level. (a) The continuous-domain representation. (b) The discrete-domain representation.

In practice, we implement the operator wavelet transform using an FFT-based algorithm [39]. Given the analytical knowledge of all filters in the Fourier domain, this implementation method allows perfect reconstruction irrespective of the filter support that may be finite or infinite. It should be noted that using the FFT should be seen as a computational method that allows deploying a fast and exact algorithm within the constraints of periodic boundary conditions.

\section{Decomposition of Elementary Singularities}

Proposition 2 (Analytical Wavelet Footprint): Analyzing an elementary singularity associated to the fractional derivative (order $\gamma_{0}$, phase parameter $\tau_{0}$, and located at position $x_{0}$ ) by the operator wavelet $\psi$ (order $\gamma$, phase parameter $\tau$ ) at scale $i$ and position $k$ leaves a footprint that can be approximated by the analytical form $2^{i\left(\gamma_{0}-1 / 2\right)} g\left(x_{0} / 2^{i}-k ; \gamma, \tau, \gamma_{0}, \tau_{0}\right)$, as defined by (24).

Proof: Given the properties of the operator wavelets and its fractional integrals, we can easily derive any wavelet coefficient $d_{i}[k]$ at scale $i$ and position $k$ of the discrete operator-wavelet decomposition for the given singularity:

$$
\begin{aligned}
d_{(i)}[k] & =\left\langle\sqrt{2^{-i}} \psi\left(\cdot / 2^{i}-k\right), \rho_{\tau_{0}}^{\gamma_{0}}\left(\cdot-x_{0}\right)\right\rangle \\
& =\sqrt{2^{-i}}\left\langle\left\{\bar{\partial}_{-\tau}^{\gamma} \phi\right\}\left(\cdot / 2^{i}-k\right), \rho_{\tau_{0}}^{\gamma_{0}}\left(\cdot-x_{0}\right)\right\rangle \\
& =2^{i\left(\gamma_{0}-1 / 2\right)}\left\langle\left\{\bar{\partial}_{\tau-\tau_{0}}^{\gamma-\gamma_{0}} \phi\right\}\left(\cdot / 2^{i}-k\right), \partial_{\tau_{0}}^{\gamma_{0}} \rho_{\tau_{0}}^{\gamma_{0}}\left(\cdot-x_{0}\right)\right\rangle \\
& =2^{i\left(\gamma_{0}-1 / 2\right)}\left\langle\left\{\bar{\partial}_{-\tau_{0}}^{-\gamma_{0}} \psi\right\}\left(\cdot / 2^{i}-k\right), \partial\left(\cdot-x_{0}\right)\right\rangle \\
& =2^{i\left(\gamma_{0}-1 / 2\right)} \underbrace{\left\{\bar{\partial}_{-\tau_{0}}^{-\gamma_{0}} \psi\right\}\left(x_{0} / 2^{i}-k\right)}_{\longrightarrow g\left(x_{0} / 2^{i}-k ; \gamma, \tau, \gamma_{0}, \tau_{0}\right)}
\end{aligned}
$$


(a)

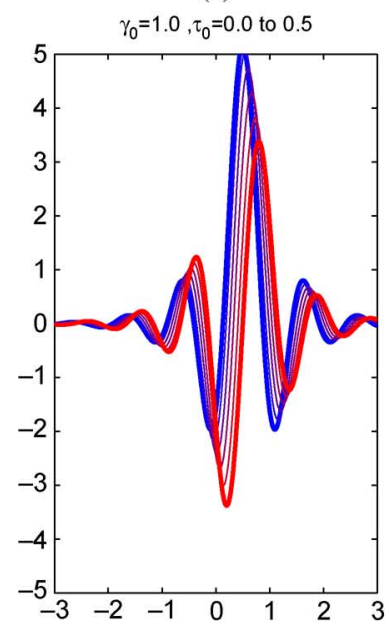

(b)

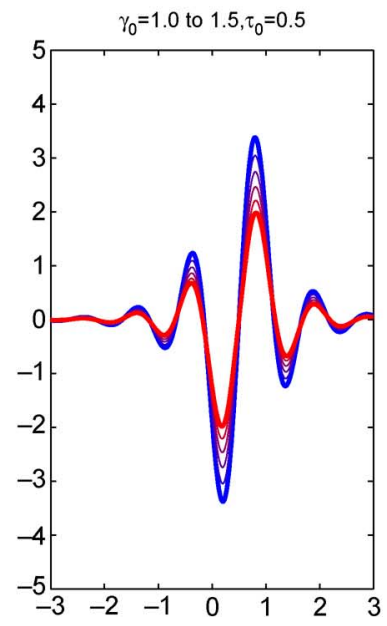

(c)

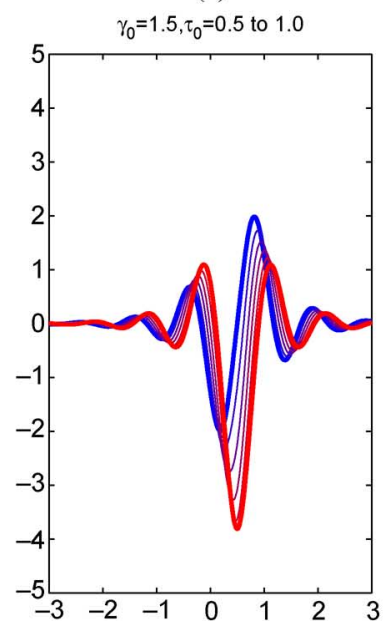

(d)

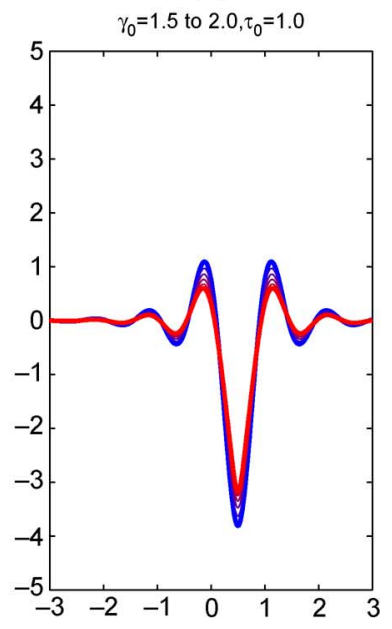

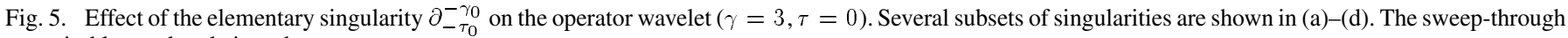
starts in blue and ends in red.

We first illustrate the analytical wavelet footprint by the nonsampled form $g\left(x ; \gamma, \tau, \gamma_{0}, \tau_{0}\right)$. In Fig. 5, the singularities of Fig. 1 are analyzed by the operator wavelet $(\gamma=3, \tau=0)$. Interestingly, changing the phase of the singularity influences the shape and symmetry of the operator wavelet, while changing the order of the singularity affects mainly the amplitude.

Proposition 3 (Lipschitz Regularity): The signal model of (8) with singularities of order $\gamma_{k}, k=1, \ldots, M$, is uniformly Lipschitz $\alpha_{0}=\max _{m}\left(\gamma_{m}-1\right)$. Consequently, there exists $A>0$ such that the coefficients $d_{(i)}[k]$ at level $i$ and position $k$ of the operator wavelet decomposition of $f$ satisfy

$$
\left|d_{(i)}[k]\right| \leq A s^{\alpha_{0}+1 / 2}
$$

where $s=2^{i}$ is the scale.

Proof: Let us introduce $\alpha_{m}=\gamma_{m}-1$ as the Lipschitz exponent of the $m$ th singularity, and the upperbound

$$
A_{m}=\max _{x}\left|g\left(x ; \gamma, \tau, \gamma_{m}, \tau_{m}\right)\right|
$$

for the limiting form of the analytical wavelet footprint. Then, using scale $s=2^{i}$, we can easily derive that

$$
\begin{aligned}
\left|d_{(i)}[k]\right| & \approx\left|\sum_{m=1}^{M} a_{m} 2^{i\left(\gamma_{m}-1 / 2\right)} g\left(x_{m} / 2^{i}-k ; \gamma, \tau, \gamma_{m}, \tau_{m}\right)\right| \\
& \leq \sum_{m=1}^{M} s^{\alpha_{m}+1 / 2}\left|a_{m}\right| \underbrace{\left|g\left(x_{m} / s-k ; \gamma, \tau, \gamma_{m}, \tau_{m}\right)\right|}_{\leq A_{m}} \\
& \leq A s^{\alpha_{0}+1 / 2}
\end{aligned}
$$

where $\alpha_{0}=\max _{m} \alpha_{m}$ and $A=M \max _{m}\left(\left|a_{m}\right| A_{m}\right)$.

When the operator wavelet (or its fractional integral) is used as a wavelet basis, the wavelet coefficients will be sampled versions of the continuous function. A useful feature is the mono- or bimodal character of these coefficients, as can be observed from Fig. 6. This property follows from the fact that the smoothing kernel-the interpolating B-spline-converges rapidly to the sinc-function [40]. Consequently, the smoothing kernel at scale 0 will have a bandwidth of approximately $[-2 \pi, 2 \pi]$. Samples of this function at positions $k \in \mathbb{Z}$ all have the same sign, while samples of its derivatives could be monoor bimodal only. This property is useful for applications, since the analytical wavelet footprint will have two local extrema at most.

\section{RESUltS AND DISCUSSION}

We now illustrate the analytical footprints for the task of retrieving elementary singularities from an operator-wavelet decomposition. The algorithm that we are using is greedy and matches footprints one-by-one in a multiscale fashion; it is summarized in Appendix D.

\section{A. Stepwise Parametric Fitting With Analytical Footprints}

To illustrate the concept, we applied the algorithm to a piecewise polynomial signal of length 512 where the singularities are discontinuities; i.e., eight singularities of the type $\rho_{0.5}^{1}$. We select the operator wavelet decomposition with parameters $\gamma=8$, $\tau=0$. In Fig. 7(a), the decomposition of the signal on the operator wavelet basis is shown with three decomposition levels. The bimodal property allows us to find potential singularity locations by looking for the local extrema $p_{m}$ at a given scale $i^{\prime}$, which are used as initial estimates $2^{i^{\prime}} p_{m}$ for the positions of the singularities. Next, we perform parametric fitting of the expression of Proposition 2 using the Levenberg-Marquardt algorithm (Matlab 7.8.0) to solve the nonlinear least-squares fitting criterion based on information at all scales. Both parameters, amplitude and edge position, are defined continuously. We use multiple initial estimates for the position (i.e., $2^{i^{\prime}}\left(p_{m}-\right.$ $\left.1), \ldots, 2^{i^{\prime}}\left(p_{m}+1\right)\right)$ and retain the parameters of the best fit. In Fig. 7(c), we show the measured and fitted coefficients for the largest local extremum of the first decomposition level (corresponding the edge at position 305 in the signal). The fitted coefficients are then subtracted from the wavelet decomposition and the procedure is repeated for the next local extremum. In this way, we obtain a list of singularities that allows us to synthetize the singularity signal shown in Fig. 7(b), together with 

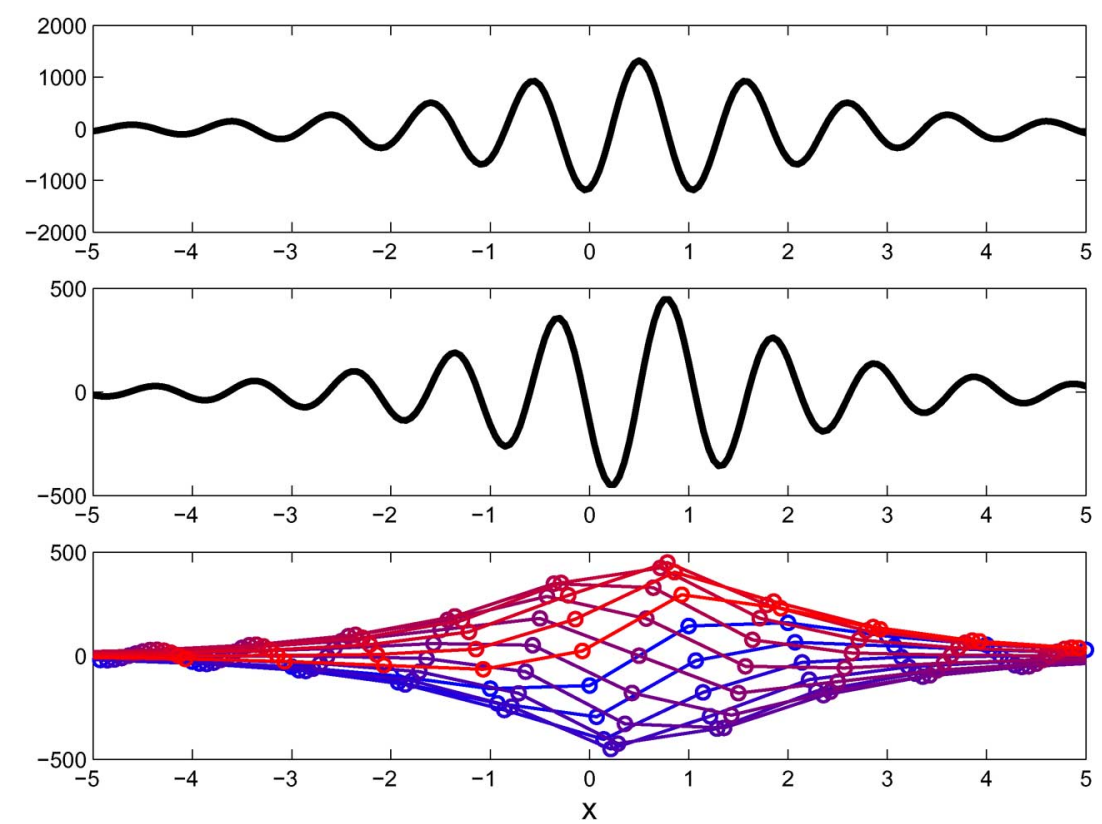

Fig. 6. Mono- or bimodal character of the wavelet coefficients of an edge singularity $\rho_{0.5}^{1}\left(x-x_{0}\right)$, where $x_{0}$ varies between 0 and 1 and 14 steps. Top: operator wavelet $\gamma=8, \tau=0$. Middle: operator wavelet acting on edge singularity. Bottom: wavelet coefficients of the edge singularity at different shifts $x_{0}$ (red to blue) are sampled versions of the middle plot; they are all mono- or bimodal.

(a)

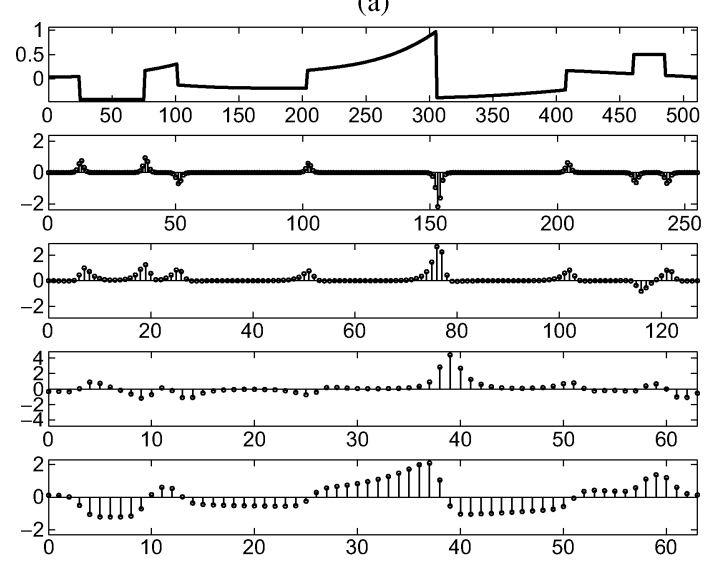

(c)

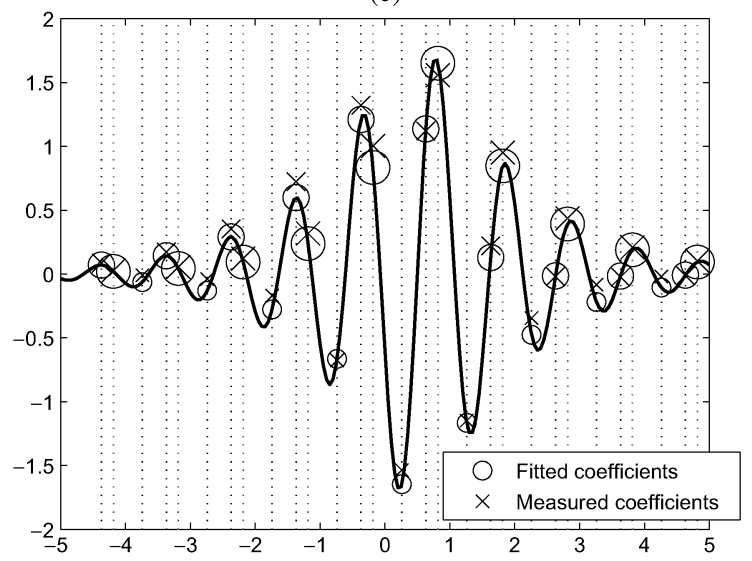

(b)
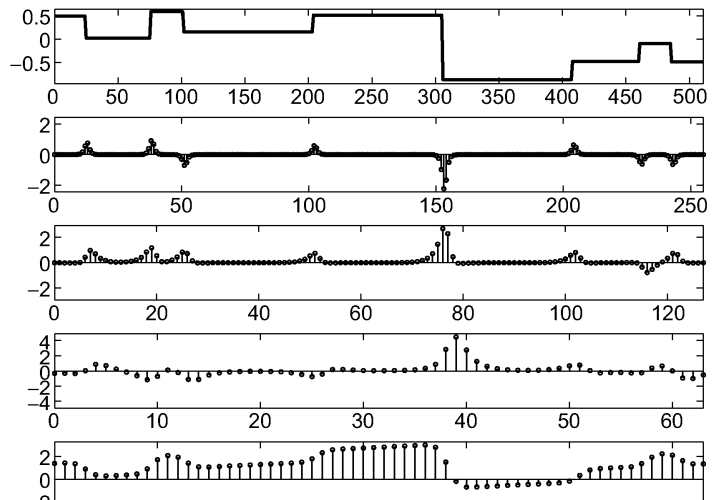
$-2-$

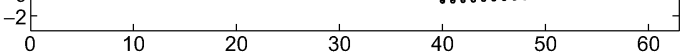

(d)

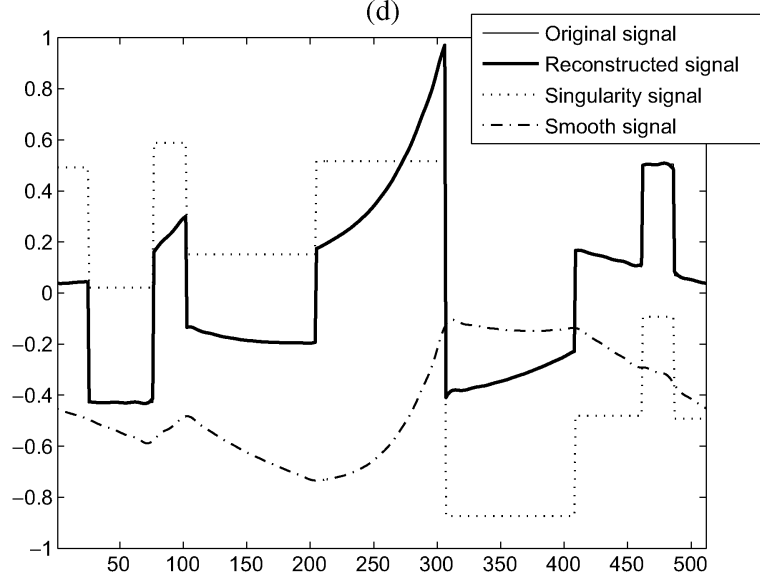

Fig. 7. (a) Original signal $\left(\gamma_{0}=1, \tau_{0}=1 / 2\right)$ with its operator wavelet decomposition. (b) Singularity signal obtained from (a) with its operator wavelet decomposition. The wavelets coefficients in (a) and (b) are almost completely identical, while the difference in the lowpass subband encodes the smooth part. (c) Result of the fitting procedure for the large edge of the signal at position 305. The fitting uses all scales at the same time; coefficients from coarse scales are larger. (d) Reconstructed signal, together with the constituting singularity and smooth signal parts. The SNR of the reconstruction is $43 \mathrm{~dB}$.

its wavelet decomposition. The wavelet subbands are identical to those of the original signal (as they should be), while the lowpass subband contains the residual that encodes the smooth polynomial part. To compensate for the asymptotic nature of 
(a)

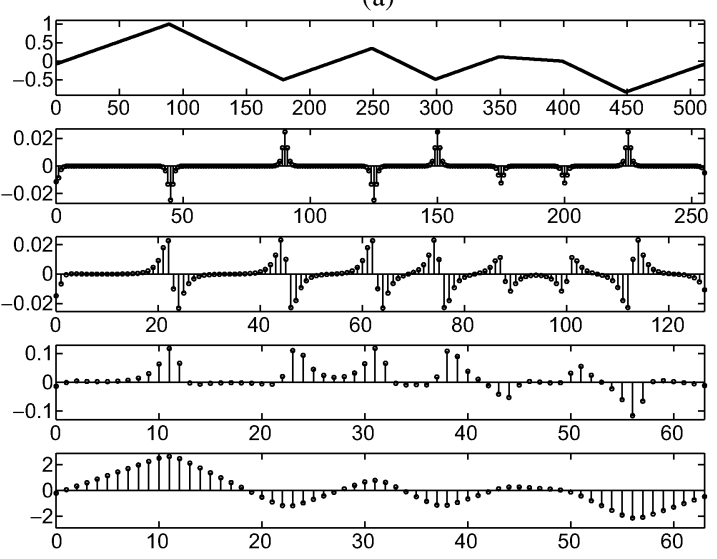

(c)

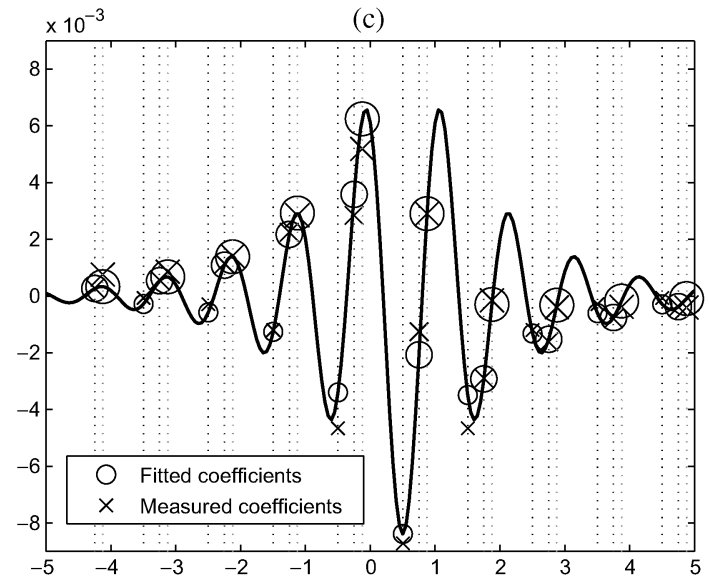

(b)
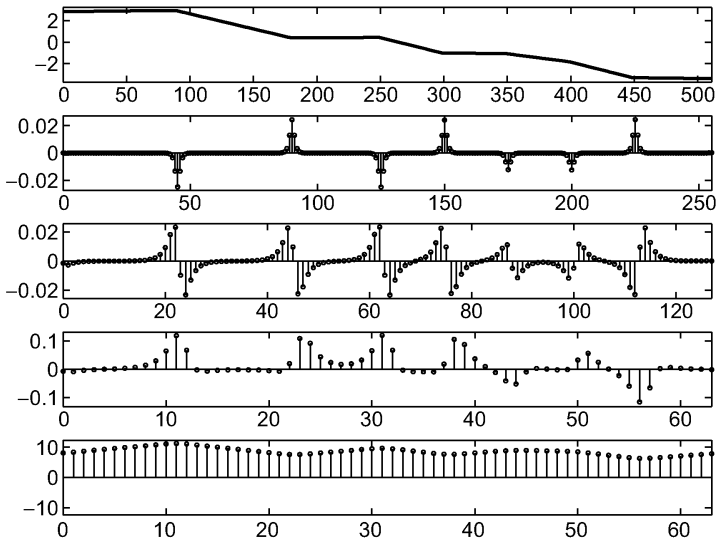

(d)

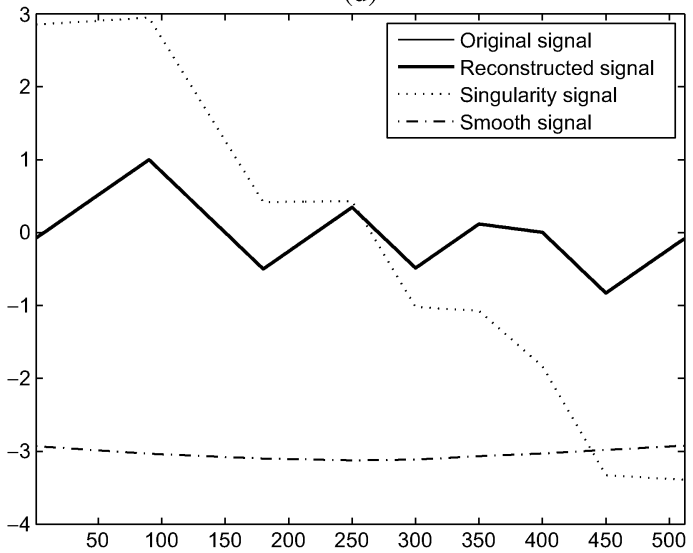

Fig. 8. (a) Original signal $\left(\gamma_{0}=2, \tau_{0}=1\right)$ with its operator wavelet decomposition. (b) Singularity signal obtained from (a) with its operator wavelet decomposition. The wavelets coefficients in (a) and (b) are almost completely identical, while the difference in the lowpass subband encodes the smooth part. (c) Result of the fitting procedure for the large edge of the signal at position 90. The fitting uses all scales at the same time; coefficients from coarse scales are larger. (d) Reconstructed signal, together with the constituting singularity and smooth signal parts. The SNR of the reconstruction is $62 \mathrm{~dB}$.

the analytical footprint, we adjust the amplitudes of the singularities by a global scaling factor obtained by linear regression between coefficients of all wavelets subbands of the synthetized singular signal and the ones of the original signal. Finally, by reconstructing the difference between the original lowpass and the one of the singularity signal, we obtain the smooth part that can be added to the singularity signal and constitutes the reconstructed signal; see Fig. 7(d). We show an example for another type of singularity (discontinuity of the derivative; i.e., $\gamma_{0}=2$, $\tau_{0}=1$ ) in Fig. 8.

One important issue is how well singularities can be separated when they are close to each other. For the proposed algorithm, singularities are fitted independently, and consequently their footprints can interfere if too close. We define the constant $L$ as the distance over which the envelope of the operator wavelet is below a given percentage $p$ of its peak value; given the sinc-like behavior of the smoothing kernel, this happens approximately for $L=(2 p)^{-1}$ (see also Fig. 6 bottom). Therefore, avoiding interaction between footprints for $J$ decomposition levels requires singularities to be spaced at least $L \cdot 2^{J}$ samples apart. Notice that this condition is very similar to the one in [15], where the size of the wavelet filter played a central role. Empirically, we found that our one-by-one fitting procedure gave correct results for $p<16 \%$ in the noiseless case, corresponding to $L>3$, which leads to a minimal signal separation of $3 \cdot 2^{J}$ for a decomposition with $J$ levels.

Finally, we mention that artificial discontinuities may arise due to the periodic boundary conditions of our FFT-based implementation. To avoid this type of artifact, we linearly detrended the singularity signal before obtaining its decomposition and then added it back to the reconstructed lowpass residual.

Although our algorithm is not specifically designed for denoising, it can yield competitive results for the class of singular signals satisfying (8). In Fig. 9, we show the outcome when applying the same algorithm as before to the signal deteriorated with additive Gaussian noise (SNR $20 \mathrm{~dB}$ ). All signal discontinuities are well recovered and the remaining noise originates from the residual lowpass subband; the total SNR improvement is about $7 \mathrm{~dB}$.

\section{B. Discussion}

We demonstrated that it is possible to extract and characterize singularities from the operator-wavelet decomposition using the analytical wavelet footprints. The analytical form replaces an explicit dictionary that needs to be learned and that suffers from redundancy in the case of shift-variant wavelet signatures (as occur with nonredundant bases). The algorithm is related to 
(a)

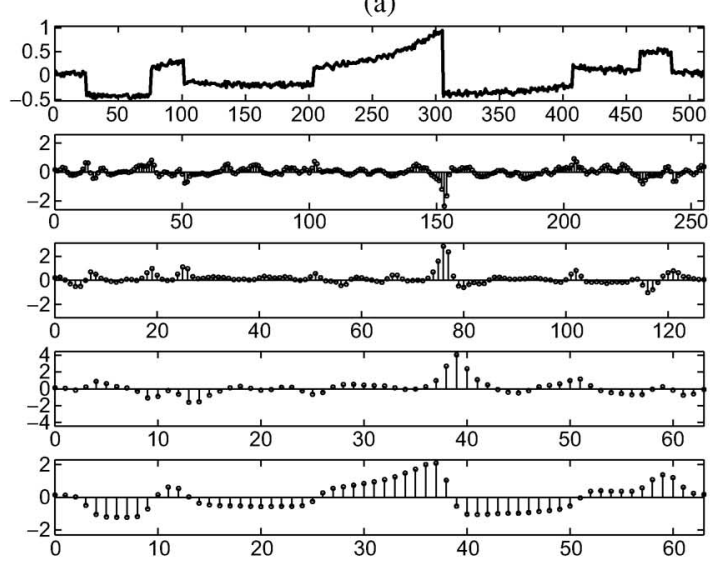

(b)

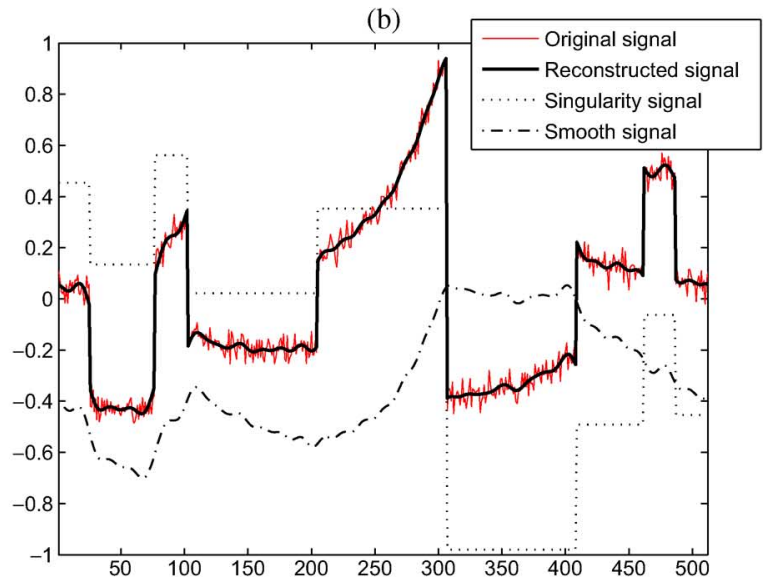

Fig. 9. The SNR improvement of the reconstructed signal is $7 \mathrm{~dB}$ (noisy signal $20 \mathrm{~dB}$ ).

matching pursuit [41] in the sense that the singularities are fitted one by one. However, the dictionary is never explicit and the parameters $\left(a_{m}, x_{m}\right)$ are retrieved by a numerical optimization algorithm. Discretizing the positions $x_{m}$ on the (uniform) measurement grid would allow to solve the problem with matching pursuit or sparsity-pursuing algorithms [3]. While applying algorithms from the discrete setting is outside the scope of this paper, theoretical results on the exact recovery criterion can shed light on the minimum spacing between singularities [42], [43]. Specifically, for the case of spike deconvolution with Morlet wavelets and small noise, the minimum spacing of $5 \cdot \tilde{s}$ was found, with $\tilde{s}$ the width (standard deviation) of the Gaussian smoothing kernel [3], [44]. For the operator wavelet, we have $\tilde{s} \approx 2^{J+1}$, which makes the bound $10 \cdot 2^{J}$. Empirically, we found a lower bound of $3 \cdot 2^{J}$ in the noiseless case, which is the same order of magnitude; the difference could be explained by high order of the operator wavelet that we use $(\gamma=8)$ and the fact that we exploit the knowledge that only two singularities needs to be fitted. Another advantage of parametric fitting of the analytical footprints is the ability to reach subsample resolution since the problem is completely formulated in the continuous domain; this opens the door to superresolution. The stepwise fitting algorithm that was applied in Section IV-A is primarily meant as a proof-of-concept. We believe that it may be improved on several counts.

In the present implementation, the singularities are fitted one after the other. A more evolved version could fit multiple singularities at the same time, explicitly taking into account their interference patterns. Another option is to rely on additional information provided by different operator wavelets. One attractive choice is to use a Hilbert-pair of operator wavelets [45], [46]; i.e., the ones corresponding to order $\gamma$ with phase parameter $\tau$, and order $\gamma$ with phase parameter $\tau+1 / 2$, respectively.

We should also mention that the approach is not necessarily optimal for denoising. Although the operator wavelet decomposition is a semi-orthogonal one, that is, wavelets of different scales are orthogonal but not within the same scale. Therefore, white noise will become correlated between wavelet coefficients at the same scale. The fitting procedure could be extended to take into account the correlation. Also, depending on the amount of noise, the local extrema could be detected at a different de- composition level or using a more robust detector. Moreover, the number of decomposition levels directly influences the amount of smoothing in the lowpass subband.

Finally, we mention that the proposed algorithm preselects the type of singularity that will be fitted. Although the same operator-wavelet decomposition can be used to deal with different singularities, going beyond this limitation is a remaining challenge for future research. Our attempts to let the parametric fitting also take care of the order or the phase of the singularities did not give satisfactory results. Another problem related to mixing signatures of singularities of different order is their significant difference in dynamic range [e.g., compare Figs. 7(a) and $8(a)]$.

\section{CONCLUSION}

In this paper, we proposed a particular wavelet basis, named operator wavelets, that is part of the family of fractional B-spline wavelets. The corresponding (nonredundant) wavelet transform acts as a multiscale fractional derivative operator, which have been studied extensively in mathematics. We provided a closed-form formula for the response of these wavelets to the whole class of self-similar $(\gamma, \tau)$ singularities. We also showed a practical algorithm to extract the singularities from a given signal and separate and reconstruct the singularity and the smooth parts. One attractive feature of our approach is that it works for nonredundant decompositions and explicitly takes into account the effect of subsampling. This could open new possibilities for signal analysis on compact decompositions.

\section{APPENDIX A}

\section{CONSTRUCTION OF FraCTIONAL B-SPLINE WAVELETS}

These B-splines satisfy the three admissibility conditions for a scaling function [17], [18]:

i) They generate a Riesz basis; i.e., the autocorrelation filter is bounded by two constants $0<C_{0} \leq\left|A\left(e^{j \omega}\right)\right| \leq C_{1}<$ $\infty$.

ii) They fulfill the partition of unity:

$$
\sum_{n} \beta_{\tau}^{\alpha}(x-n)=1 .
$$

iii) They satisfy a two-scale relation. 
Consequently, the B-splines generate a multi-resolution analysis of $L_{2}(\mathbb{R})$; that is, a sequence of nested subspaces

$$
\cdots \subset \mathcal{V}_{2} \subset \mathcal{V}_{1} \subset \mathcal{V}_{0} \subset \mathcal{V}_{-1} \subset \mathcal{V}_{-2} \subset \cdots \subset L_{2}(\mathbb{R}) .
$$

We now revisit and extend the design strategy for the semiorthogonal spline wavelet transform that was initially proposed in [31]. To that end, we select the wavelet space

$$
\mathcal{W}_{i}=\operatorname{span}_{n \in \mathbb{Z}}\left\{\sqrt{2^{-i}} \psi\left(\frac{x}{2^{i}}-n\right)\right\}
$$

as the orthogonal complement of $\mathcal{V}_{i}$, such that $\mathcal{V}_{i} \oplus \mathcal{W}_{i}=\mathcal{V}_{i-1}$. The wavelet is therefore constrained to be orthogonal to all integer-shifted versions of the scaling function at the same scale, which also implies orthogonality to wavelets at different scales; i.e., $\mathcal{W}_{i} \perp \mathcal{W}_{j}$, for $i \neq j$.

By construction, the wavelet at scale 1 is included in $\mathcal{V}_{0}$, which is expressed as

$$
\psi\left(\frac{x}{2}\right)=\sqrt{2} \sum_{k} w[k] \beta_{\tau}^{\alpha}(x-k) .
$$

It also has to fulfill the orthogonality condition $\mathcal{V}_{1} \perp \mathcal{W}_{1}$ : b

$$
\left\langle\psi\left(\frac{\dot{2}}{2}\right), \beta_{\tau}^{\alpha}(\dot{\overline{2}}-n)\right\rangle=0, \quad n \in \mathbb{Z} .
$$

Taking the $z$-transform of (31) results into

$$
W(z) H\left(z^{-1}\right) A(z)+W(-z) H\left(-z^{-1}\right) A(-z)=0 .
$$

This condition requires $W(z) H\left(z^{-1}\right) A(z)$ to be an odd polynomial; thus the degree of freedom of $Q\left(z^{2}\right)$.

\section{APPENDIX B}

\section{ProOF OF PROPOSITION 1}

First, we verify that the proposed form of $W(z)$ satisfies the semi-orthogonality condition of (32). We then derive the Riesz bounds of the wavelet filter as

$$
\begin{aligned}
R\left(e^{j \omega}\right) & \\
= & \sum_{k}|\hat{\psi}(\omega+2 \pi k)|^{2} \\
= & \frac{1}{2}\left|W\left(z^{1 / 2}\right)\right|^{2} A\left(z^{1 / 2}\right)+\frac{1}{2}\left|W\left(-z^{1 / 2}\right)\right|^{2} A\left(-z^{1 / 2}\right) \\
= & |Q(z)|^{2} A\left(z^{1 / 2}\right) A\left(-z^{1 / 2}\right) \\
& \times\left(\frac{\left|H\left(-z^{-1 / 2}\right)\right|^{2}}{2} A\left(-z^{1 / 2}\right)+\frac{\left|H\left(z^{1 / 2}\right)\right|^{2}}{2} A\left(z^{1 / 2}\right)\right) \\
= & |Q(z)|^{2} A\left(z^{1 / 2}\right) A\left(-z^{1 / 2}\right) A(z) .
\end{aligned}
$$

Consequently, the wavelet generates a Riesz basis if $0<C_{0} \leq$ $\left|Q\left(e^{j \omega}\right)\right| \leq C_{1}<\infty$. This guarantees a stable perfect reconstruction filter bank when implementing the discrete wavelet transform.

To characterize the operator-like behavior of the semi-orthogonal spline wavelet, we rewrite (18) as

$$
\begin{aligned}
& 2 \hat{\psi}(2 \omega) \\
& \quad=\sqrt{2} W\left(e^{j \omega}\right) \hat{\beta}_{\tau}^{\alpha}(\omega)
\end{aligned}
$$

$$
\begin{aligned}
& =\sqrt{2} e^{-j \omega} Q\left(e^{j 2 \omega}\right) H\left(-e^{-j \omega}\right) A\left(-e^{j \omega}\right) \cdot \frac{\hat{\Delta}_{\tau}^{\gamma}\left(e^{j \omega}\right)}{\hat{\partial}_{\tau}^{\gamma}(\omega)} \\
& =\hat{\partial}_{\tau}^{\gamma}(-\omega) \sqrt{2} e^{-j \omega} \frac{Q\left(e^{j 2 \omega}\right) H\left(-e^{-j \omega}\right) A\left(-e^{j \omega}\right)}{\hat{\Delta}_{\tau}^{\gamma}\left(e^{-j \omega}\right)} \frac{\hat{\Delta}_{0}^{2 \gamma}\left(e^{j \omega}\right)}{\hat{\partial}_{0}^{2 \gamma}(\omega)} \\
& =\hat{\partial}_{\tau}^{\gamma}(-\omega) 2^{-\alpha} e^{-j \omega} Q\left(e^{j 2 \omega}\right) A\left(-e^{j \omega}\right) \hat{\beta}_{0}^{2 \gamma-1}(\omega)
\end{aligned}
$$

where we have used the identity $H(-z)=2^{-\alpha-(1 / 2)} \hat{\Delta}_{\tau}^{\alpha+1}(z)$. Note that in (34), none of the factors except $\hat{\partial}_{\tau}^{\gamma}(-\omega)$ cancels at $\omega=0$. Therefore, we derive the asymptotic form of (34) as

$$
\mathcal{F}\{\psi(\cdot / 2)\}(\omega)=C_{2} e^{-j \omega} \hat{\partial}_{\tau}^{\gamma}(-\omega)+O\left(|\omega|^{\gamma+1}\right), \text { as } \omega \rightarrow 0
$$

with the constant $C_{2}=\lim _{\omega \rightarrow 0} 2^{-\alpha} Q\left(e^{j \omega}\right) A\left(-e^{j \omega}\right)=$ $\left(2^{-\gamma+2} / \pi^{2 \gamma}\right) \zeta(2 \gamma)\left(2^{2 \gamma}-1\right) Q(1)$, where $\zeta(s)=\sum_{n>0}\left(1 / n^{s}\right)$ is the Riemann zeta function.

\section{APPENDIX C \\ PROOF OF PROPOSITION 1}

First, we show that the operator wavelet of (22) is obtained for $Q\left(z^{2}\right)=2^{\alpha} /(A(z) A(-z))$, which is bounded and thus a valid choice. For this $Q\left(z^{2}\right)$, we find in the Fourier domain that

$$
2 \hat{\psi}(2 \omega)=\hat{\partial}_{\tau}^{\gamma}(-\omega) e^{-j \omega} \frac{\hat{\beta}_{0}^{2 \gamma-1}(\omega)}{A\left(e^{j \omega}\right)} .
$$

The term $1 / A\left(e^{j \omega}\right)$ can be recognized as the interpolation prefilter of the $2 \gamma$ th order symmetric B-spline. By identifying the interpolating B-spline $\beta_{\text {int }}^{2 \gamma-1}$, we further develop (36) as

$$
2 \hat{\psi}(2 \omega)=\hat{\partial}_{\tau}^{\gamma}(-\omega) e^{-j \omega} \hat{\beta}_{\text {int }}^{2 \gamma-1}(\omega)
$$

which corresponds in the spatial domain to

$$
\psi\left(\frac{x}{2}\right)=\bar{\partial}_{\tau}^{\gamma} \beta_{\mathrm{int}}^{2 \gamma-1}(x-1) .
$$

The resulting wavelet filter becomes

$$
W(z)=z^{-1} 2^{\alpha} \frac{H\left(-z^{-1}\right)}{A(z)}
$$

which can be rewritten as

$$
W(z) \frac{1}{\hat{\Delta}_{\tau}^{\gamma}\left(z^{-1}\right)} A(z)=z^{-1}
$$

and thus satisfies the wavelet filter condition derived in Appendix A.

Second, the expression of the operator wavelet (36) can be further manipulated in the Fourier domain:

$$
2 \hat{\psi}(2 \omega)=\hat{\partial}_{\tau}^{\gamma}(-\omega) \frac{e^{-j \omega}}{1+|\omega|^{2 \gamma} \sum_{k \neq 0} \frac{1}{|\omega+2 \pi k|^{2 \gamma}}} .
$$

The proposed wavelet depends on the space defined by the B-spline scaling function, but is independent of the choice of a particular spline scaling function. Since the second factor in (41) is essentially constant for $\omega \leq \pi$, we may infer that this wavelet closely matches the frequency response of the operator over a large range in the Fourier domain. Indeed, the order of 
the second term of the Taylor development at $\omega=0$ increased from $\gamma+1$ to $3 \gamma$ :

$$
\hat{\psi}(\omega) \propto \hat{\partial}_{\tau}^{\gamma}(-\omega / 2) e^{-j \omega / 2}+O\left(|\omega|^{3 \gamma}\right), \quad \text { as } \omega \rightarrow 0 .
$$

Finally, we derive an explicit form of $\partial_{-\tau_{0}}^{-\gamma_{0}} \psi$. We start from (41) and use the fact that the wavelet $\hat{\psi}(2 \omega)$ has most of its energy mostly around $\pi$ and $-\pi$. This allows us to approximate the sum in the denominator by two dominant terms

$$
\begin{aligned}
& \frac{2 \hat{\psi}(2 \omega)}{(-j \omega)^{\gamma_{0} / 2-\tau_{0}}(j \omega)^{\gamma_{0} / 2+\tau_{0}}} \\
& \approx \frac{e^{-j \omega}(j \omega)^{\left(\gamma-\gamma_{0}\right) / 2-\left(\tau+\tau_{0}\right)}(-j \omega)^{\left(\gamma-\gamma_{0}\right) / 2+\left(\tau+\tau_{0}\right)}}{1+\left|\frac{\omega}{\omega-2 \pi}\right|^{2 \gamma}+\left|\frac{\omega}{\omega+2 \pi}\right|^{2 \gamma}} .
\end{aligned}
$$

We now focus on the positive frequencies $\omega>0$ and apply the change of variables $\omega=\pi(1+\eta)$ where $\eta$ is close to 0 :

$$
\begin{aligned}
& \hat{\partial}_{-\tau_{0}}^{-\gamma_{0}} 2 \hat{\psi}^{+}(2 \omega) \\
& =\frac{e^{-j \pi(1+\eta)} \pi^{\gamma-\gamma_{0}}(-j(1+\eta))^{\frac{\gamma-\gamma_{0}}{2}-\tau-\tau_{0}}(j(1+\eta))^{\frac{\gamma-\gamma_{0}}{2}+\tau+\tau_{0}}}{1+\left|\frac{1+\eta}{1-\eta}\right|^{2 \gamma}+\left|\frac{1+\eta}{3+\eta}\right|^{2 \gamma}} \\
& \approx \frac{e^{-j \pi(1+\eta)} \pi^{\gamma-\gamma_{0}} e^{-j \pi\left(\tau+\tau_{0}\right)}(1+\eta)^{\gamma-\gamma_{0}}}{1+\left|\frac{1+\eta}{1-\eta}\right|^{2 \gamma}} \\
& \approx \frac{e^{-j \pi(1+\eta)} \pi^{\gamma-\gamma_{0}} e^{-j \pi\left(\tau+\tau_{0}\right)} e^{\left(\gamma-\gamma_{0}\right) \eta}}{1+e^{4 \gamma \eta}}
\end{aligned}
$$

where the last step makes use of the approximation $1+\eta \approx e^{\eta}$. This latter expression we then use to compute the Fourier inverse of the analytical signal of the wavelet:

$$
\begin{aligned}
\partial_{-\tau_{0}}^{-\gamma_{0}} \psi^{+}(x) & \frac{1}{2 \pi} \int_{-\infty}^{+\infty} \hat{\partial}_{-\tau_{0}}^{-\gamma_{0}} \hat{\psi}^{+}(\omega) e^{j \omega x} d \omega \\
= & \frac{1}{\pi} \int_{-\infty}^{+\infty} \hat{\partial}_{-\tau_{0}}^{-\gamma_{0}} \hat{\psi}^{+}(2 \omega) e^{j 2 \omega x} d \omega \\
\approx & -\frac{\pi^{\gamma-\gamma_{0}} e^{-j \pi\left(\tau+\tau_{0}\right)}}{2} \int_{-\infty}^{+\infty} \frac{e^{\left(\gamma-\gamma_{0}\right) \eta}}{1+e^{4 \gamma \eta}} e^{j \pi(2 x-1)(1+\eta)} d \eta \\
= & -\frac{\pi^{\gamma-\gamma_{0}} e^{-j \pi\left(\tau+\tau_{0}-2 x\right)}}{8 \gamma} \\
& \times \underbrace{\int_{0}^{1} \xi^{-\frac{\gamma-\gamma_{0}}{4 \gamma}-j \frac{\pi(2 x-1)}{4 \gamma}}(1-\xi)^{-1+\frac{\gamma-\gamma_{0}}{4 \gamma}+j \frac{\pi(2 x-1)}{4 \gamma}} d \xi}_{0} \\
= & -\frac{\left.\pi^{\gamma-\gamma_{0}+1} e^{j \pi\left(2 x-\left(\tau+\tau_{0}\right)\right)}, \frac{\gamma-\gamma_{0}}{4 \gamma}+j \frac{\pi(2 x-1)}{4 \gamma}+j \frac{\pi(2 x-1)}{4 \gamma}\right)}{8 \gamma \sin \left(\frac{\pi\left(\gamma-\gamma_{0}\right)}{4 \gamma}+j \frac{\pi^{2}(2 x-1)}{4 \gamma}\right)}
\end{aligned}
$$

where we used the change of variables $1 /\left(1+e^{4 \gamma \eta}\right)=\xi$ to identify the Beta function for which the identity $B(1-a, a)=$ $\Gamma(1-a) \Gamma(a)=\pi / \sin (a \pi)$ holds. This ultimately yields the following asymptotic expression for the operator wavelet and its fractional integrals:

$$
\partial_{-\tau_{0}}^{-\gamma_{0}} \psi(x) \approx-\frac{\pi^{\gamma-\gamma_{0}+1}}{4 \gamma} \operatorname{Re}\left[\frac{e^{j \pi\left(2 x-\left(\tau+\tau_{0}\right)\right)}}{\sin \left(\frac{\pi\left(\gamma-\gamma_{0}\right)}{4 \gamma}+j \frac{\pi^{2}(2 x-1)}{4 \gamma}\right)}\right] .
$$

The complex argument of the sin-function can be evaluated using $\sin (a+j b)=\sin (a) \cosh (b)+j \cos (a) \sinh (b)$.

\section{APPENDIX D}

\section{Algorithm FOR STEPWISE PARAMETRIC FITTING}

We first choose the operator wavelet transform (order $\gamma$, phase $\tau$ ). Next, we select the singularity type that we want to recover (order $\gamma_{0}<\gamma$, phase $\tau_{0}$ ). The signal $f$ is decomposed in the operator wavelet basis, resulting in coefficients $d_{(i)}[k]$ at scales $i=1, \ldots, J+1$ and positions $k$. For a chosen subband at scale $i^{\prime}$, we now make a list of the local extrema $p_{m}, m=1, \ldots, M$. The core of the algorithm will now fit singularities one by one for each local extremum. Specifically, we optimize the parameters of the analytical footprint (amplitude $a_{m}$, position $x_{m}$ ) using the Levenberg-Marquardt optimization algorithm based on the multiscale information of the wavelet coefficients $d_{(i)}[k], i=1, \ldots, J$. We try several initial estimates for the position $x_{m}: 2^{i^{\prime}}\left(p_{m}-1\right), \ldots, 2^{i^{\prime}}\left(p_{m}+1\right)$. Next, the fitted analytical footprint is subtracted from the coefficients $d_{(i)}[k](i=1, \ldots, J)$. After fitting $M$ singularities, we synthetize the singularity signal $f_{1}$ using $a_{m} \rho_{\tau_{0}}^{\gamma_{0}}\left(x-x_{m}\right)$ and obtain its wavelet coefficients $d_{(i)}^{\prime}[k]$. We now adjust the singularity amplitudes $a_{m}$ by least-squares solution of the optimal scaling factor between $d_{(i)}^{\prime}[k]$ and the initial coefficients $d_{(i)}[k]$. Finally, we reconstruct the smooth signal $f_{0}$ from the residual lowpass $c_{(J)}[k]-c_{(J)}^{\prime}[k]$ only. Our approximation of $f$ based on the singular signal model is given by $f_{1}+f_{0}$.

\section{REFERENCES}

[1] S. Mallat, "A theory for multiresolution signal decomposition: The wavelet decomposition," IEEE Trans. Pattern Anal. Mach. Intell., vol. 11, no. 7, pp. 674-693, 1989.

[2] I. Daubechies, "The wavelet transform, time-frequency localization and signal analysis," IEEE Trans. Inf. Theory, vol. 36, no. 5, pp. 961-1005, 1990 .

[3] S. Mallat, A Wavelet Tour of Signal Processing: The Sparse Way. San Diego, CA: Academic, 2009.

[4] S. Mallat, "Zero-crossings of a wavelet transform," IEEE Trans. Inf. Theory, vol. 37, no. 4, pp. 1019-1033, Jul. 1991.

[5] S. Mallat and S. Zhong, "Characterization of signals from multiscale edges," IEEE Trans. Pattern Anal. Mach. Intell., vol. 14, no. 7, pp. 710-732, Jul. 1992.

[6] S. Mallat and W. L. Hwang, "Singularity detection and processing with wavelets," IEEE Trans. Inf. Theory, vol. 38, no. 2, pp. 617-643, 1992.

[7] Y.-P. Wang, "Image representations using multiscale differential operators," IEEE Trans. Image Process., vol. 8, no. 12, pp. 1757-1771, 1999.

[8] J. F. Clearbout and F. Muir, "Robust modeling of erratic data," Geophys., vol. 38, no. 5, pp. 826-844, 1973.

[9] F. Santosa and W. W. Symes, "Linear inversion of band-limited reflection seismograms," SIAM J. Sci. Statist. Comput., vol. 7, no. 4, pp. 1307-1330, 1986.

[10] F. J. Herrmann, "Singularity characterization by monoscale analysis: Application to seismic imaging," Appl. Comput. Harmon. Anal., vol. 11, pp. 64-88, 2001 
[11] M. Maysami, "Lithology constraints from seismic waveforms: Application to Opal-A to Opal-CT transition," Ph.D. dissertation, Dept. of Earth and Ocean Sciences, Univ. of British Columbia, Vancouver, BC, Canada, 2008.

[12] F. J. Herrmann, "Seismic deconvolution by atomic decomposition: A parametric approach with sparseness constraints," Integr., vol. 12, no. 1, pp. 69-91, 2005.

[13] C. Li, C. Zheng, and C. Tai, "Detection of ECG characteristic points using wavelet transforms," IEEE Trans. Biomed. Eng.bb, vol. 42, no. 1, pp. 21-28, 1995.

[14] B. M. Sadler and A. Swami, "Analysis of multiscale products for step detection and estimation," IEEE Trans. Inf. Theory, vol. 45, no. 3, pp. 1043-1051, Apr. 1999.

[15] P. L. Dragotti and M. Vetterli, "Wavelet footprints: Theory, algorithms, and applications," IEEE Trans. Signal Process., vol. 51, no. 5, pp. 1306-1323, 2003.

[16] V. Bruni and D. Vitulano, "Wavelet based signal denoising via simple singularities approximation," Signal Process., vol. 86, pp. 859-876, 2006.

[17] M. Unser and T. Blu, "Fractional splines and wavelets," SIAM Rev., vol. 42, pp. 43-67, 2000.

[18] T. Blu and M. Unser, "A complete family of scaling functions: The $(\alpha, \tau)$-fractional splines," in Proc. 28th IEEE Int. Conf. Acoustics, Speech, Signal Processing (ICASSP'03), Hong Kong, Apr. 2003, vol. VI, pp. 421-424.

[19] K. S. Miller and B. Ross, An Introduction to the Fractional Calculus and Fractional Differential Equations. New York: Wiley, 1993.

[20] S. G. Samko, A. A. Kilbas, and O. I. Marichev, Fractional Integrals and Derivative. Theory and Applications. New York: Gordon and Breach, 1993.

[21] I. Podlubny, Fractional Differential Equations. New York: Academic, 1998.

[22] A. A. Kilbas, H. M. Srivastava, and J. J. Trujillo, Theory and Applications of Fractional Differential Equations. Philadelphia, PA: Saunders, 2006.

[23] F. Mainardi, Fractional Calculus and Waves in Linear Viscoelasticity: An Introduction to Mathematical Models. Singapore: World Scientific, 2010.

[24] R. Gorenflo and F. Mainardi, "Fractional calculus: Integral and differential equations of fractional order," in Fractals and Fractional Calculus in Continuum Mechanics. New York: Springer-Verlag, 1997, vol. 378, CISM Courses and Lectures, pp. 223-276.

[25] M. D. Ortigueira, "On the fractional central differences and derivatives,” J. Vibrat. Control, vol. 14, no. 9-10, pp. 1255-1266, 2008.

[26] M. Unser and T. Blu, "Self-similarity: Part I-Splines and operators," IEEE Trans. Signal Process., vol. 55, no. 4, pp. 1352-1363, Apr. 2007.

[27] I. M. Gel'fand and G. E. Shilov, Properties and Operations Transl.: E. Saletan. New York: Academic, 1964, vol. I, Generalized Functions.

[28] L. Schwartz, Théorie des Distributions. Paris, Franceb: Hermann, 1998.

[29] D. C. Champeney, A Handbook of Fourier Theorems. Cambridge, U.K.: Cambridge Univ. Press, 1987.

[30] T. Blu and M. Unser, "Wavelets, fractals, and radial basis functions," IEEE Trans. Signal Process., vol. 50, no. 3, pp. 543-553, Mar. 2002.

[31] M. Unser, A. Aldroubi, and M. Eden, "A family of polynomial spline wavelet transforms," Signal Process., vol. 30, pp. 141-162, 1993.

[32] G. Battle, "A block spin construction of ondelettes. Part I: Lemarié functions," Commun. Math. Phys., vol. 110, pp. 601-615, 1987.

[33] C. K. Chui and J.-Z. Wang, "A cardinal spline approach to wavelets," Proc. Amer. Math. Soc., vol. 113, no. 3, pp. 785-793, Nov. 1991.

[34] C. Micchelli, C. Rabut, and F. I. Utreras, "Using the refinement equation for the construction of pre-wavelets III: Elliptic splines," Numer. Algorithms 1, no. 4, pp. 331-352, 1991.

[35] I. Khalidov, D. Van De Ville, T. Blu, and M. Unser, "Construction of wavelet bases that mimic the behaviour of some given operator," in Proc. SPIE Conf. Mathematical Imaging: Wavelet XII, San Diego, CA, Aug. 26-29, 2007, vol. 6701, pp. 67010S-1-67010S-7.

[36] D. Van De Ville and M. Unser, "Complex wavelet bases, steerability, and the Marr-like pyramid," IEEE Trans. Image Process., vol. 17, no. 11, pp. 2063-2080, Nov. 2008.

[37] M. Unser, D. Sage, and D. Van De Ville, "Multiresolution monogenic signal analysis using the Riesz-Laplace wavelet transform," IEEE Trans. Image Process., vol. 18, no. 11, pp. 2402-2418, Nov. 2009.

[38] M. Unser and D. Van De Ville, "Higher-order Riesz transforms and steerable wavelet frames," in Proc. 2009 IEEE Int. Conf. Image Processing (ICIP), Cairo, Egypt, Nov. 7-11, 2009, pp. 3801-3804.
[39] T. Blu and M. Unser, "The fractional spline wavelet transform: Definition and implementation," in Proc. 25th IEEE Int. Conf. Acoustics, Speech, Signal Processing (ICASSP), Istanbul, Turkey, Jun. 5-9, 2000, vol. I, pp. 512-515.

[40] A. Aldroubi, M. Unser, and M. Eden, "Cardinal spline filters: Stability and convergence to the ideal sinc interpolator," Signal Process., vol. 28, no. 2, pp. 127-138, Aug. 1992.

[41] S. Mallat and Z. Zhang, "Matching pursuit with time-frequency dictionaries," IEEE Trans. Signal Process., vol. 41, no. 12, pp. 3397-3415, 1993.

[42] J. A. Tropp, "Greed in good: Algorithmic results for sparse approximation," IEEE Trans. Inf. Theory, vol. 50, no. 10, pp. 2231-2242, 2004.

[43] R. Gribonval and M. Nielsen, "Beyond sparsity: Recovering structured representations by $\ell^{1}$ minimization and greedy algorithms," $A d v$. Comput. Math., vol. 28, no. 1, pp. 23-41, 2008.

[44] C. Dossal and S. Mallat, "Sparse spike deconvolution with minimum scale," presented at the SPARS05, Rennes, France, Nov. 16-18, 2005.

[45] N. Kingsbury, "Complex wavelets for shift invariant analysis and filtering of signals," Appl. Comput. Harmon. Anal., vol. 10, no. 3, pp. 234-253, May 2001.

[46] K. N. Chaudhury and M. Unser, "Construction of Hilbert transform pairs of wavelet bases and Gabor-like transforms," IEEE Trans. Signal Process., vol. 57, no. 9, pp. 3411-3425, Sep. 2009.

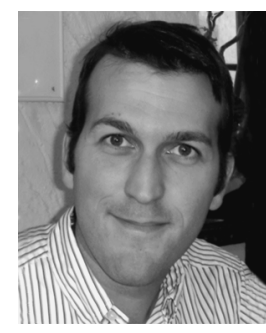

Dimitri Van De Ville (M'02) received the M.S degree in engineering and computer sciences and the Ph.D. degree from Ghent University, Belgium, in 1998, and 2002, respectively.

He received a grant as a Research Assistant with the Fund for Scientific Research Flanders Belgium (FWO). In 2002, he joined Prof. M. Unser's Biomedical Imaging Group at the École Polytechnique Fédérale de Lausanne (EPFL), Switzerland. In December 2005, he became responsible for the Signal Processing Unit at the University Hospital of Geneva, Switzerland, as part of the Centre d'Imagerie Biomédicale (CIBM). In 2009, he has been the recipient of a Swiss National Science Foundation professorship and currently holds a joint position at the University of Geneva, Switzerland, and the EPFL. His research interests include wavelets, sparsity, pattern recognition, and their applications in biomedical imaging, such as functional magnetic resonance imaging.

Dr. Van De Ville served as an Associate Editor for the IEEE TRANSACTIONS ON IMAGE PROCESSING from 2006 to 2009 and the IEEE SignAL PROCESSING LETTERS from 2004 to 2006. He is a member of the Bio Imaging and Signal Processing (BISP) TC of the IEEE Signal Processing Society. Since 2003, he has also been an Editor and Webmaster of The Wavelet Digest. He is a Guest Co-Editor of the 2011 Special Issue on Brain Decoding in Pattern Recognition $\mathrm{He}$ is Co-Chair of the 2007, 2009, and 2011 Wavelets series conferences, together with V. Goyal and M. Papadakis.

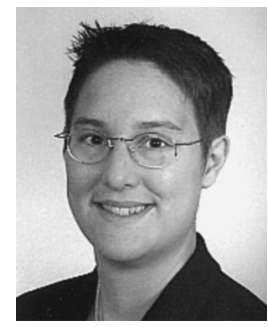

Brigitte Forster-Heinlein (M'04) received the Diploma and Ph.D. degrees (summa cum laude) in mathematics from the Technische Universität München (TUM), Germany, in 1998 and 2001, respectively.

From 2002 to 2004 , she was a Researcher in the Biomedical Imaging Group under M. Unser at the Swiss Federal Institute of Technology (EPFL), Lausanne, Switzerland. Since 2005, she has been a "Junior Professor" of mathematical modeling in biomedical engineering at TUM. From 2005 to 2009 , she additionally was team leader of the Marie Curie Excellence Team MAMEBIA, funded by the European Commission, at the Helmholtz Zentrum MünchenGerman Research Center for Environmental Health. Her main research topics are approximation theory, sampling theory, complex splines, multiresolution and wavelets, and applications for signal and image processing.

Dr. Forster is a Member of the Editorial Board of the International Journal for Mathematics and Mathematical Sciences (IJMMS). 


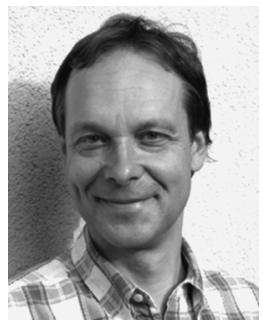

Michael Unser (M'89-SM'94-F'99) received the M.S. (summa cum laude) and Ph.D. degrees in electrical engineering from the École Polytechnique Fédérale de Lausanne (EPFL), Switzerland, in 1981 and 1984, respectively.

From 1985 to 1997 , he was a Scientist with the National Institutes of Health, Bethesda, MD. He is currently a Full Professor and Director of the Biomedical Imaging Group, EPFL. His main research area is biomedical image processing. He has a strong interest in sampling theories, multiresolution algorithms, wavelets, and the use of splines for image processing. He has published over 150 journal papers on those topics.

Dr. Unser has held the position of associate Editor-in-Chief for the IEEE TRANSACTIONS ON MEDICAL IMAGING from 2003 to 2005 and has served as Associate Editor for the same journal from 1999 to 2002 and from 2006 to 2007, the IEEE TRANSACtions ON IMAGE PROCESSING from 1992 to 1995, and the IEEE SignAl PROCESSING LETTERS from 1994 to 1998. He co-organized the First IEEE International Symposium on Biomedical Imaging (ISBI) 2002. He was the founding Chair of the Technical Committee of the IEEE Signal Processing Society on Bio Imaging and Signal Processing (BISP). He received the 1995 and 2003 Best Paper Awards, the 2000 Magazine Award, and the 2008 Technical Achievement Award from the IEEE Signal Processing Society. He is one of ISIs Highly Cited Authors in Engineering (http://isihighlycited.com). $\mathrm{He}$ is a EURASIP Fellow and a member of the Swiss Academy of Engineering Sciences.

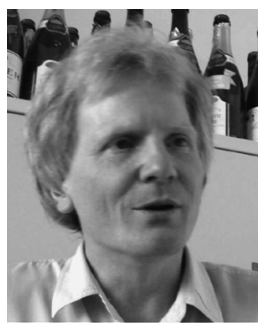

Thierry Blu (M'96-SM'06) was born in Orléans, France, in 1964. He received the Diplôme d'lingénieur degree from École Polytechnique, France, in 1986 and from Télécom Paris (ENST), France, in 1988, and the Ph.D. degree in electrical engineering from ENST in 1996 for a study on iterated rational filter banks, applied to wideband audio coding.

Between 1998 and 2007, he was with the Biomedical Imaging Group at the Swiss Federal Institute of Technology (EPFL), Lausanne, Switzerland. Currently, he is a Professor in the Department of Electronic Engineering, The Chinese University of Hong Kong. His Research interests focus on (multi)wavelets, multiresolution analysis, multirate filter banks, interpolation, approximation and sampling theory, sparse sampling, image denoising, psychoacoustics, biomedical imaging, optics, and wave propagation.

Dr. Blu was the recipient of two Best Paper Awards from the IEEE Signal Processing Society in 2003 and 2006. He is also coauthor of a paper that received a Young Author Best Paper Award in 2009 from the same society. Between 2002 and 2006, he was an Associate Editor for the IEEE TRANSACTIONS ON IMAGE PROCESSING, and, since 2006, he has been an Associate Editor for the IEEE TRANSACtions on Signal Processing. He is also Associate Editor of Elsevier's Signal Processing the and EURASIP Journal on Image and Video Processing. 